\title{
Reshaping the Gut Microbiome in An Apc-Mutant Genetic Background: Mechanistic Insights From Integrated Multi-Omics
}

\section{Ying-Shiuan Chen}

The University of Texas MD Anderson Cancer Center

Jia Li

Texas A\&M University System Health Science Center College of Medicine: Texas A\&M University College of Medicine

\section{Rani Menon}

Texas A\&M University College Station

\section{Arul Jayaraman}

Texas A\&M University College Station

\section{Kyongbum Lee}

Tufts University

\section{Yun Huang}

Texas A\&M University System Health Science Center College of Medicine: Texas A\&M University College of Medicine

\section{Wan Mohaiza Dashwood}

Texas A\&M University System Health Science Center College of Medicine: Texas A\&M University College of Medicine

\section{Ke Zhang}

Texas A\&M University System Health Science Center College of Medicine: Texas A\&M University College of Medicine

\section{Deqiang Sun}

Texas A\&M University System Health Science Center College of Medicine: Texas A\&M University College of Medicine

\section{Roderick Dashwood ( $\square$ rdashwood@tamu.edu )}

Texas A\&M University Health Sciences Center https://orcid.org/0000-0003-0351-4034

\section{Research}

Keywords: Microbiome, transcriptome, metabolome, anticancer mechanisms, spinach 
DOl: https://doi.org/10.21203/rs.3.rs-143373/v1

License: (c) (1) This work is licensed under a Creative Commons Attribution 4.0 International License. Read Full License 


\section{Abstract}

Background: Complex interrelationships govern the dynamic interactions between gut microbes, the host, and exogenous drivers of disease outcome. A multi-omic approach to cancer prevention by spinach was pursued in the polyposis in rat colon (Pirc) model of colorectal cancer.

Results: Spinach fed for 26 weeks ( $10 \%$ w/w, freeze-dried in the diet) exhibited significant antitumor efficacy in the Pirc model and, despite the Apc-mutant genetic background, no changes were detected in b-catenin protein expression. To provide mechanistic leads, we performed 16S rRNA sequencing of the gut microbiome and unbiased transcriptomic analyses of colon polyps and matched normal-looking tissues. For dietary spinach intervention groups, increased microbiome diversity coincided with reversal of taxonomic composition in both wild type and Apc-mutant rats. Metagenomic prediction implicated linoleate and butanoate metabolism, tricarboxylic acid cycle, and pathways in cancer, which was supported by mechanistic leads from metabolomic analyses. Specifically, anticancer outcomes were linked to several spinach-derived linoleate bioactives with known anti-inflammatory and proapoptotic mechanisms, as well as $\mathrm{N}$-aceto-2-hydroxybutanoate changes that were consistent with altered butanoate metabolism stemming from the increased a-diversity of the gut microbiome. On the other hand, L-glutamate and $\mathrm{N}$-acetylneuraminate were reduced markedly in colon tumors from spinach-fed rats, implicating deregulated mitochondrial energetics and altered cell surface glycans involved in oncogenic signaling networks, immune evasion, and other pathways in cancer.

Conclusions: Tumor suppression by dietary spinach was observed for the first time in the Apc-mutant Pirc model, and was independent of the mechanistic targeting of b-catenin. Marked reshaping of the gut microbiome in spinach-fed rats occurred along with changes in host transcriptomics and RNA-miRNA networks. Metabolomics provided mechanistic support for linoleate and butanoate metabolism, which are both strongly linked to anticancer outcomes in the colon. Tumor-associated changes in L-glutamate and $\mathrm{N}$-acetylneuraminate warrant further investigation in the context of synthetic lethality and circumventing deregulated signaling networks in colorectal cancer patients.

\section{Background}

The mammalian gut microbiome is strongly implicated in host physiology and pathophysiology [1-3]. For example, studies in germ-free models of colorectal cancer (CRC) revealed decreased bowel inflammation and tumor outcomes as compared with the corresponding animals under conventional housing conditions $[4,5]$. Fecal microbiota transplantation was used successfully to treat recurrent Clostridium difficile infection [6], and provided benefit to patients with inflammatory bowel diseases, functional gastrointestinal disorders, and obesity [7].

There is increasing interest in defining interventions that alter the gut microbiota for disease prevention and treatment. Epidemiological studies indicate that CRC is associated with low consumption of green vegetables and fiber, whereas intake of dark leafy vegetables is linked to decreased risk [8]. However, little 
is known about how these dietary intakes influence the crosstalk between gut microbiota, host transcriptomics, and pathogenesis in the gastrointestinal tract.

Spinach is a dark leafy vegetable with a high chlorophyll content and bioactive phytochemicals such as betaine, carotenoids, flavonoids, and polyunsaturated fatty acids [9-11]. We employed an adenomatous polyposis coli (Apc)-mutant rat model [12-15] to examine anticancer outcomes from dietary spinach consumption. The polyposis in rat colon (Pirc) model mimics disease progression in human familial adenomatous polyposis patients, involving spontaneous tumor development both in the colon and in the small intestine [15,16]. The genetic model circumvents the need for carcinogen treatment, used previously with dietary spinach [17], and the burden of adenomatous polyps facilitates temporal tracking of tumor suppression via colonoscopy $[12,14,18]$.

We observed significant antitumor efficacy from dietary spinach consumption and, despite the Apcmutant genetic background, b-catenin protein levels remained highly overexpressed in colon polyps. Subsequently, mechanisms were pursued linking gut microbiome to host multi-omic changes in fatty acid metabolism, the tricarboxylic acid (TCA) cycle, and pathways in cancer.

\section{Methods}

\section{Preclinical}

Prior approval was obtained from the Institutional Animal Care and Use Committee. After weaning, Pirc (F344/NTac-Apc ${ }^{a m 1137}$, Taconic Farms, Inc. USA) and wild type (WT) F344 male rats were assigned randomly to basal AIN93 control diet (Ctrl) or AIN93 diet containing $10 \% \mathrm{w} / \mathrm{w}$ freeze-dried spinach by weight (SPI). Diets were replenished every 2 to 3 days, and animal body weights were monitored weekly. Monthly endoscopy was employed for temporal tracking of polyp development in the rat colon, as described before [12,14]. Each polyp was assigned a unique 'address' in the colon, based on a reported methodology [18]. Prior to termination, 3-4 rats in each group were injected with $\mathrm{BrdU}$ (100 mg/kg body weight), and animals were euthanized $1 \mathrm{~h}$ later by $\mathrm{CO}_{2}$ inhalation. A thorough necropsy examination was performed, and tissue samples were taken for histopathology and molecular analysis, as reported $[12,14]$.

\section{Histology}

Tissue sections ( $5 \mathrm{~mm}$ ) were stained with hematoxylin and eosin (H\&E), or immunostained with antibodies for b-catenin (BD Biosciences \# 610153), cleaved caspase-3 (Cell signaling \#9661), and BrdU (BD Biosciences \# 347580), at the Research Histology, Pathology and Imaging Core, The University of Texas MD Anderson Cancer Center. A BrdU labeling index was determined, as described [61], and cleaved caspase-3 was quantified as percent positive-stained crypts in a given field. Two to three biological replicates were employed for WT-Ctrl, WT-SPI, Pirc-Ctrl, and Pirc-SPI groups. For each tissue section, at least 15 independent fields were quantified in the proximal, middle, and distal regions of the colon. Other proteins were immunoblotted as reported [12-14,62-65], using Cell Signaling primary antibodies for PARP 
(\#9542), b-catenin (\#9581), p53 (\#9282), and cyclin D1 (\#2926), with b-actin as loading control (Sigma, A1978).

\section{Microbiome}

Frozen gut contents from rats in each group were submitted for bacterial genomic DNA extraction at the Center for Metagenomics and Microbiome Research (CMMR), Baylor College of Medicine, Houston, TX, followed by microbiome analyses as reported [66-68]. The 16S rDNA V4 region was amplified and barcoded via PCR and sequenced using the MiSeq platform (Illumina) with the 2x250 bp paired-end protocol. OTUs at a similarity cutoff value of $97 \%$ were generated by the UPARSE algorithm and mapped to SILVA database. OTU tables and Agile Toolkit for Incisive Microbial Analyses (ATIMA) were provided by CMMR for primary data visualization. ATIMA microbiome data were subjected to the Kruskal-Wallis test, with the adjusted P-values indicated in the figures. LEfSe used rarefied OTU data from CMMR, and results were generated with LDA $>2$ and $p<0.05$ (Kruskal-Wallis test). PICRUSt used raw BIOM files to map Greengenes for metagenome prediction, coupled to Statistical Analysis of Metagenomic Profiles (STAMP). Results were replotted using Prism or Excel. To associate microbiome and disease outcome, bacterial diversity and OTUs were integrated with matched tumor multiplicity and tumor volume datasets. At the family and genus level, tumor outcomes were plotted by linear regression analysis, coupled to Pearson correlation coefficient. To integrate microbiome OTUs with host gene predisposition and anticancer outcomes, we calculated the Pearson correlation by group for enriched microbiome OTUs from LEfSe (genus level) and the reads per kilo base per million mapped reads (RPKM) of the host transcriptome.

\section{Transcriptome}

Flash-frozen rat colon tissues ( $\mathrm{n}=3$ replicates per group) were subjected to total RNA extraction using a Qiagen miRNeasy mini kit, according to the manufacturer's instructions. After DNase digestion and purity/integrity confirmation, as reported [12,14], RNA samples were run on a $1 \%$ denaturing agarose gel prior to library preparation for RNA and small RNA sequencing. For RNA library preparation, NEBNext® Poly(A) mRNA Magnetic Isolation Module (NEB, E7490) and NEBNext ${ }^{\circledR}$ Ultra ${ }^{\text {TM }}$ Directional RNA Library Prep Kit (NEB, E7420) were performed with $3 \mathrm{mg}$ RNA input as the starting material. For small RNA library

preparation, NEBNext ${ }^{\circledR}$ Small RNA Library Prep Set for Illumina ${ }^{\circledR}$ (NEB, E7300) were performed with 1 mg RNA input as the starting material. The RNA went through 3' ligation, primer hybridization, $5^{\prime}$ ligation, first strand synthesis, PCR amplification and size selection (MinElute Gel Extraction Kit, Qiagen). During library construction, each sample was tagged with a specific index at the PCR amplification step. The library concentration was measured by Qubit dsDNA HS Assay Kit (Thermo Fisher Scientific) and the size of the library was assessed by $2 \%$ agarose gel with $2 \mathrm{ng}$ library material. The samples were diluted to $1 \mathrm{pmol}$ for further library pooling. Before loading the pooled library into the NextSeq 550/500 system (Illumina), 1 $\mathrm{nM}$ library was denatured and diluted according to NextSeq System Denature and Dilute Libraries Guide (Illumina). Stranded RNA sequencing was conducted by paired-end sequencing with 75-bp read length 
and small RNA sequencing was conducted by single-end sequencing with $50 \mathrm{bp}$ read length. Fastq files were generated on BaseSpace for further bioinformatics processing and analysis.

\section{Metabolome}

Pre-weighed samples of rat colon tumor and normal colonic mucosa ( $n=6-7$ biological replicates), collected at the time of necropsy, were homogenized in $0.5 \mathrm{ml}$ cold methanol and $0.2 \mathrm{ml}$ chloroform in pre-cooled Garnet bead tubes using a Precellys $\AA 24$ beadbeater. Samples were centrifuged at $3000 \mathrm{rpm}$ for 10 mins at $4^{\circ} \mathrm{C}$ and $0.7 \mathrm{ml}$ cold water was added to the supernatant. The aqueous phase was collected by centrifugation at $3000 \mathrm{rpm}$ for $1 \mathrm{~min}$, and the extraction procedure was repeated. The pooled aqueous (upper) phase was passed through a sterile nylon cell strainer and lyophilized (Labconco ${ }^{\mathrm{TM}}$ ). Lyophilized samples were reconstituted in $50 \mathrm{ml}$ methanol/water $(1: 1, \mathrm{v} / \mathrm{v})$ and stored at $-80^{\circ} \mathrm{C}$ until analysis. Untargeted liquid chromatography high-resolution accurate-mass spectrometry (LC-HRAM ${ }^{\text {TM }}$ ) profiling was conducted on a Q Exactive ${ }^{T M}$ Plus Hybrid Quadrupole-Orbitrap ${ }^{T M}$ Mass Spectrometer coupled to a Dionex UltiMate 3000 high-performance liquid chromatography system (Thermo Scientific ${ }^{\mathrm{TM}}$ ). A Synergi Fusion-RP C-18 column (Phenomenex) was used with a methanol/acetonitrile solvent gradient, and mass scanning in the positive mode was in the range 50 to 750. The MS1 and MS1-dependent MS2 spectra were collected at an $\mathrm{m} / \mathrm{z}$ resolution of 70,000 and 17,500 , respectively, with the autosampler maintained at $4^{\circ} \mathrm{C}$. Methanol/water $(1: 1 \mathrm{~V} / \mathrm{V})$ blanks were injected between each run to prevent sample carryover. Parallel studies also were conducted on the freeze-dried spinach.

Raw metabolomic data were imported into Progenesis QI (Waters) for alignment, peak picking, and compound identification. Among the 17243 features detected, candidates were identified by reference to the Human Metabolome Database (HMDB) and KEGG. Raw abundance data were normalized to initial sample weights, incorporating Partial Least Squares Discriminant Analysis (PLSDA). Features were further filtered by their appearance in three independent metabolomic databases, with at least three biological replicates and a significant ANOVA test. This resulted in 5946 differential features for further analysis. Significant features were subjected to clustering and correlation by MetaboAnalyst 4.0 [69-72]. The $\mathrm{p}$-values (two-tailed $t$-test) and $t$-scores (standardized test statistic) were generated for multiple group comparisons of metabolic networks and functional metabolite prediction via Mummichog version 2 in $R$ [73]. Primary prediction of 883 compound names was mapped to the KEGG COMPOUND Database, and pathway analyses by Mummichog were ranked according to the $p$-value, using $p=0.05$ as the cutoff.

\section{Bioinformatics}

RNA-seq data were processed and analyzed as reported [12]. DEGs were called using DESeq2, with adjusted $p$-value $<0.05$. MiRNA-seq raw fastq data were trimmed using cutadaptor, and reads with the same sequence were collapsed and counted. Mature and hairpin miRNA sequence data for rat were downloaded from miRbase (http://www.mirbase.org/ftp.shtml). Collapsed reads were mapped to rat mature miRNA sequences using blastn. The counts table was input to DESeq2 to call DEmiRs with adjusted $p$-value $<0.05$. PCA was performed using DESeq2 for both RNA-seq and miRNA-seq datasets. 
DEGs were further analyzed by GSEA (Java Desktop application v3.0 Beta 2), with a cutoff of FDR<25\%. Six miRNAs were prioritized for target RNA analysis. Target prediction was conducted by Targetscan 7.2 for rat specific analysis, and miRNA-mRNA pairs with p-value $<-0.5$ were retained in Cytoscape [74]. Additional methodologies were as reported [75-78].

\section{Quantitative PCR}

Basic methodologies for RNA extraction and purification were as reported, using three biological replicates per group [12-14,79-81]. After SuperScript III (Thermo Fisher Scientific) or miScript RT II (Qiagen) kits, quantitative PCR (qPCR) reactions were performed by LightCycler ${ }^{\circledR}$ FastStart DNA Master SYBR Green (Roche Applied Science) on a LightCycler96 instrument. Primers for mRNA qPCR were designed by NCBI-BLAST (see Table 11 in Additional file 3), whereas primers for miRNA qPCR were purchased custom-made from Qiagen. Internal controls were Gapdh and U6B small nuclear RNA for mRNA and miRNA analyses, respectively. RNAs and miRNAs were selected based on prior validation and ranking in GSEA data. Original gene lists for tight junction and anti-microbial function were obtained from KEGG and Gene Ontology (GO) resources, respectively, and further sorted by the sequencing data to generate genes of interest. We focused on six miRNAs consistently altered in Pirc colon tumors, and filtered miRNA-mRNA pairs by conserved UTR target site in human and rat, with a linear correlation $<-0.7$ in sequencing data. Verification of miRNAs and mRNAs was by qPCR (Pearson's test, with $r<-0.5$ ).

\section{Statistics}

Statistical analysis of two-group comparisons was performed using an unpaired two-tailed $t$-test. Correlation analysis was performed by linear and Pearson's correlation, for miRNA-RNA target and microbiome-host correlations, in tumor and diet intervention groups. For matched tumor outcomemicrobiome correlation analysis, seven biological replicates of Pirc/Ctrl or Pirc/SPI were undertaken, whereas three biological replicates were used for SPI responsive gene-microbiome correlations. Unbiased metabolomic analyses used $n=6-7$ biological replicates per group. In the figures, each datapoint designates a single colon tumor or normal colonic mucosa sample from individual rats in the corresponding groups. One-way ANOVA was used to compare the mean of each column with the mean of every other column, with Tukey correction for multiple comparisons (GraphPad Prism 9.0). The level of significance was designated in the figures as follows: ${ }^{*} p<0.05 ;{ }^{\star \star} p<0.01 ;{ }^{\star \star \star} p<0.001$; ${ }^{* \star \star \star} p<0.0001$, or with the exact $p$ value [80-84].

\section{Results}

\section{Antitumor efficacy of dietary spinach in an Apc-mutant rat model}

Pirc and wild-type (WT) rats were fed AIN93 control (Ctrl) diet, or AIN93 diet containing 10\% w/w freezedried baby spinach (SPI), starting at 4 weeks of age (Fig. 1a). No significant treatment-related effects were observed with respect to food consumption and body weight throughout the study (Fig. 1b). By colonoscopy $[12,14,18]$, SPI suppressed adenomatous polyps as early as 12 weeks into the experiment 
(Fig. 1c), i.e., after 8 weeks of SPI treatment. During this period and at later times, colonoscopy data revealed consistent inhibition of small colon polyps, and significant suppression of large colon polyps after week 20 (Fig. 1d).

When the experiment was terminated, after the rats had reached 30 weeks of age, tumor multiplicity was decreased significantly both in the colon and in the small intestine, and tumor volume also was reduced significantly in the colon by SPI treatment (Fig. 1e). No marked changes were observed histologically, but bromodeoxyuridine (BrdU) labeling hinted at reduced cell proliferation rates by SPI in some regions of the colonic crypt (Additional file 1). Immunohistochemistry and immunoblotting experiments indicated that bcatenin overexpression in colon tumors was unaffected by SPI treatment (Fig. $1 \mathrm{f}$ and Additional file 2). Thus, despite the oncogenic driver of the Apc-mutant genetic background, antitumor mechanisms other than b-catenin downregulation were pursued.

\section{The gut microbiota is altered by dietary spinach}

We performed 16S rRNA sequencing of the gut microbial community in Pirc and WT rats. For a complete view of the taxonomic and other data, refer to Additional file 3. The observed Operational Taxonomic Units (OTUs, Table 1 in Additional file 3) and Shannon index revealed that a-diversity was unaffected by host genotype, but was increased significantly by SPI treatment in Pirc and WT rats (Fig. 2a, black vs. green symbols). There was no segregation between Pirc and WT rats for weighted UniFrac principal coordinates analysis (PCoA) (Fig. 2b), but a significant separation was observed in unweighted UniFrac PCoA (Fig. 2c). The gut microbiome in both genotypes clustered separately in weighted UniFrac PCoA between Ctrl and SPI groups (Fig. 2b), with a marked shift in unweighted UniFrac PCoA (Fig. 2c). These data are consistent with previous findings indicating that diet plays a dominant role over genetic background with respect to shaping interindividual variations in host-associated microbial communities $[19,20]$.

The Pirc model had a higher abundance of Bacteroidetes and Proteobacteria than WT rats, while Firmicutes, Actinobacteria, and Tenericutes were lower (Fig. 2d), as observed in mouse and human microbiomes [21-24]. These abundances were reversed by SPI intake, independent of host genotype, as evidenced by the increased relative abundance of Firmicutes and decreased Bacteroidetes (Fig. 2e). Similar findings were noted at the Family (Fig. 2f) and genus level (Fig. 2g). For example, in Pirc and WT rats, SPI treatment increased the relative abundance of Lachnospiraceae and decreased Ruminococcaceae (Fig 2f, green bars), and at the genus level SPI ingestion reduced the relative abundance of Bacteroides and Desulfovibrio (Fig. 2g, green bars). These results suggested that SPI consumption reshapes the microbiome composition, reversing the effects of the Apc-mutant background and host genetic predisposition.

Linear discriminant effect size (LEfSe) was used to further analyze the OTU microbiome data (Tables 2-4 in Additional file 3). From the corresponding cladograms (Fig. 3a-c), host genotype and dietary SPI intake both influenced Ruminococcaceae and Lachnospiraceae family members. In response to SPI treatment, LEfSe analyses revealed that Pirc and WT rats shared $~ 50 \%$ commonality among changes at the genus 
level (Fig. 3d). Phylogenetic Investigation of Communities by Reconstruction of Unobserved States (PICRUSt) categorized 328 terms following Kyoto Encyclopedia of Genes and Genomes (KEGG) analysis (Table 5 in Additional file 3). Linoleate and ether lipid metabolism were altered significantly in Pirc vs. WT rats, and PICRUSt revealed a marked effect of SPI intake. Thus, after SPI consumption, 85 terms (57 decreased and 28 increased) and 112 terms (71 decreased and 41 increased) were changed in Pirc and WT rats, respectively, and seventy-five terms overlapped between the two genotypes, i.e., 54 decreased and 21 increased (Fig. 3e). Increases in membrane transporters, cell motility, signal transduction, transcription, carbohydrate metabolism, and kinases were among the top 10 terms prioritized by KEGG analysis in Pirc and WT rats given SPI, along with porphyrin and chlorophyll metabolism (Fig. 3f). Decreased terms were related to protein translation, replication/repair, and energy/nucleotide metabolism. Pathway changes included an increase in linoleate and butanoate metabolism, and a decrease in the TCA cycle and pathways in cancer (Fig. 3g).

\section{Spinach consumption impacts key genes associated with pathogenesis}

RNA-sequencing (RNA-seq) mapped 17,378 transcripts in colonic tissues from Pirc and WT rats, and principal component analysis (PCA) completely segregated tumor tissues from normal tissues (Fig. 4a). Tumor development had a more marked effect on overall transcriptome levels than host genetics (Pirc vs. WT rats) and SPI consumption. There were 261 differentially expressed genes (DEGs) identified between Pirc and WT normal-looking tissues, half of which (138 genes) overlapped with 2180 DEGs associated with tumor development (Fig. 4b). Heatmaps of all DEGs showed a distinct tumor feature when compared to Pirc and WT normal-looking tissues (Fig. 4c, PCT vs. PCN and PCT vs. WCN).

Geneset Enrichment Analysis (GSEA) combined with HALLMARK identified nine pathways altered significantly in Pirc normal-looking colon compared to WT normal colon (Fig. 4d, upper panel), indicating differences at the level of host genetics. Five of these pathways were further altered in colon tumors (Fig. 4d, lower panel, underlined), i.e., Inflammatory Response, Allograft Rejection, TNFa signaling through NF$\mathrm{kB}$, Adipogenesis, and Myogenesis. As expected in the Apc-mutant background, Wnt/b-catenin signaling was upregulated in Pirc colon tumors (Fig. 4d, red font). Immunoblotting corroborated that b-catenin overexpression was associated with poly(ADP-ribose)polymerase (PARP) cleavage, increased cyclin D1, and decreased p53 in Pirc tumors compared to adjacent normal and WT normal colonic tissues (Additional file 2).

Other pathways of note were related to cell cycle changes, immune response, oxidative stress, and metabolism (Fig. 4d). Using RT-qPCR for validation, genes upregulated significantly in Pirc colon tumors vs. Pirc adjacent normal-looking colon included Cxcl6, Serpine1, and II-1b, whereas Hspb8, Tpm2 and Fh/1 were downregulated significantly (Fig. 4e). Compared to adjacent normal colon, upregulation of Defa6 and $B c / 3$ and downregulation of $A p p, M y h 11$, and $M y / 9$ indicated changes in tight junctions and antimicrobial activity in Pirc colon tumors (Fig. 4f).

We also mapped 559 microRNAs (miRNAs) via small RNA-seq, which segregated tumor vs. normallooking colon (Fig. 4g). Similar to the mRNA profiles (Fig. 4c), miRNAs had a distinct tumor feature as 
compared to Pirc and WT normal-looking tissues (Fig. 4h, PCT vs. PCN and PCT vs. WCN). There were 115 differentially expressed miRNAs (DEmiRs) associated with tumor formation (Fig. $4 \mathrm{~h}$ and Table 6 in Additional file 3). We combined TargetScan with RNA-seq and small RNA-seq datasets to identify miRNARNA pairs most altered in the Apc-mutant background (Fig. 4i). Validation by qPCR corroborated significant downregulation in Pirc colon tumors of miR-215, miR-143, and mir-145 compared with adjacent normal-looking colonic mucosa (Fig. 4j). Other candidates, such as mir-146b, mir-34a, and mir21 , did not reach statistical significance.

Attention shifted next to SPI effects on predicted targets (Fig. 5 and Additional file 4). Compared to the AIN basal diet control group, SPI consumption altered 4 genes in common among the 101 DEGs in WT rats and 80 DEGs in Pirc normal colon (Fig. 5a). GSEA indicated significant downregulation of cell cyclerelated pathways and upregulation of immune-related pathways in Pirc and WT rats fed SPI, with five pathways in common among the genotypes (Fig. 5b). The latter pathways included TNFa Signaling through NFkB, Hypoxia, Epithelial Mesenchymal Transition, Apoptosis, and KRAS Signaling. After SPI consumption, 2945 DEGs were identified in Pirc colon tumors compared to adjacent normal colon, and 1754 of the DEGs also were detected in tumors vs. adjacent normal colon from rats given control diet (Fig. 5c). Among the pathways most strongly implicated were IFN-a and IFN-g for tumors from SPI-fed rats compared to rats given control diet.

We also considered two scenarios for the antitumor efficacy: (1) genes up- or downregulated in colon tumors relative to adjacent normal colon that were reversed by SPI in Pirc normal colon, and (2) genes that were normalized in colon tumors from rats given SPI compared with colon tumors from Pirc rats given control diet. The first scenario would implicate primary prevention of colonic aberrant crypt foci or microadenomas, before they advanced to later stages. These genes included Serpine1, Itga6,Duoxa2, Tcf $7 / 1$, Plcd1 and S/c30a10 (Fig. 5d). Comparing tumor to tumor in scenario 2, Ccl21 and KIf7 were normalized by SPI ingestion, relative to basal diet (Fig. 5e).

In terms of miRNAs, among 66 DEmiRs in colon tumors from SPI-fed rats, 41 DEmiRs similarly were detected in colon tumors from animals on Ctrl diet (Fig. 5f). After investigating RNA-miRNA pairs and validating as before (Fig 4j), colon tumors had loss of miR-145 with increased Serpine1 and gain of mir34a with reduced $\mathrm{KIf4}$ (Fig. $5 \mathrm{~g}$ and Table 7 in Additional file 3). A negative correlation for mir145/Serpine1 was maintained after SPI consumption, whereas the mir-34a/KIf4 trend was reversed by SPI treatment (Fig. 5g).

\section{Crosstalk between microbiome and host transcriptome responses}

Integrating antitumor outcomes (Fig. 1) with a-diversity (Fig. 2), we observed a significant inverse association for tumor multiplicity (Fig. 6a, left panel) but not tumor volume (Fig. 6a, right panel). Tumor multiplicity was inversely correlated with three unclassified Bacteroidales families (Fig. 6b and Table 8 in Additional file 3). At the genus level, one unclassified Lachnospiraceae and one unclassified Ruminococcaceae genus were negatively correlated with tumor multiplicity, whereas one other unclassified Ruminococcaceae genus was positively correlated (Fig. 6c). Metagenome prediction in 
relation to tumor multiplicity outcomes found significant inverse correlations for butanoate metabolism and calcium signaling, and positive associations for peptidases and pathways in cancer (Fig. 6d).

We also compared microbiome and host gene expression changes based on the transcriptomic data (Fig. 6e). Significant positive correlations were noted for Lachnospiraceae (Unc0396i) and the efflux transporter S/c30a10, Bacteroidales (Unc00krl) and Ruminococcaceae (Unc01k4o) and the phospholipase $\mathrm{C}$ family member P/cd1, and Ruminococcaceae (Unide781 and Unc00vst) and the serine protease inhibitor Serpine1. Negative correlations were detected for Ruminococcaceae (Unide781 and Unc00vst) and the transcription factor Tcf7/1.

\section{Metabolomic corroboration of mechanistic leads}

To validate correlations from the microbiome and transcriptome studies, metabolomics was performed on adenomatous colon polyps and normal colon tissues obtained from Pirc rats at 30 weeks (Fig. 1a). As predicted from the microbiome data (Fig. 3g), among the fifty-one metabolites identified (Table 9 in Additional file 3) several were associated with fatty acid metabolism, the TCA cycle, and pathways in cancer (Fig. 7). Linoleate and its downstream metabolites from the 15-lipoxygenase-1 (15-LOX-1) pathway exert proapoptotic antitumor mechanisms in CRC [25-27]; notably, lower levels of these metabolites in Pirc colon tumors tended to be normalized in adenomatous polyps following SPI treatment, comparable to the levels detected in normal-looking Pirc colon \pm SPI (Fig. 7a). Similar trends were observed for 2-aceto-2-hydroxybutanoate, which was increased significantly in colon tumors after SPI treatment (Fig. 7b). On the other hand, L-glutamate and $\mathrm{N}$-acetylneuraminate were detected at higher levels in colon tumors, and SPI treatment reduced these metabolites in adenomatous polyps, comparable to the levels observed in normal-looking Pirc colon \pm SPI (Figs. 7c and 7d). Key intermediates are discussed further below.

\section{Discussion}

We examined the interrelationships between host genetics, gut microbial composition, dietary exposure, and disease outcome in an Apc-mutant preclinical model that mimics hereditary human CRC [12-15]. A lower diversity in the gut microbiome was circumvented by feeding dietary SPI under conditions in which significant suppression of adenomatous polyps occurred in the colon and small intestine. This work extends prior observations on the decreased microbial diversity and loss of Firmicutes, Clostridia, and Lachnospiraceae in $\mathrm{Apc}^{\mathrm{Min} /+}$ mouse [21] and human colorectal cancers [28,29]. In a short-term clinical study, Firmicutes was decreased by consumption of an animal-based diet, whereas Bacteroidetes was lowered by a plant-based diet [20]. Ruminococcaceae phylotypes were increased by resistant starch in obese men, whereas Lachnospiraceae phylotypes were increased by non-starch polysaccharides [30]. These trends were recapitulated in the current investigation, with decreasing Bacteroidetes and increasing Firmicutes and Lachnospiraceae after intervention with dietary SPI. Spinach consumption also diminished Desulfovibrio sulfur-reducing bacteria, implicating a role for anti-inflammatory responses and enhanced gut barrier function [31,32]. 
In contrast to the marked effect on the gut microbiome, long-term SPI intake exerted a relatively mild impact on host transcriptomics, based on mRNA and miRNA sequencing. We prioritized genes associated with adenomatous polyp suppression by SPI that were correlated with microbiome abundance. Serpine1 encodes plasminogen activator inhibitor type 1 (PAl-1), which is elevated in sporadic and hereditary CRC, and has an essential role in extracellular matrix proteolysis and matrix metalloproteinase activity [33-35]. Mucosal gene expression profiling of SERPINE1, plus inflammatory regulators such as CXCL 1, STAT3, and $I L$ family members, revealed associations with the decreased abundance of Firmicutes and Bacteroidetes subsets, as in human CRC [36]. DUOXA2 is a maturation factor for the epithelial antimicrobial dual oxidase DUOX2, which is among several NADPH oxidase/dual-oxidase family members deregulated in CRC and Crohn's disease, acting via NFkB [37,38]. DUOX2 expression was negatively correlated with Bacteroides, Lachnospiraceae, and Blautia, but positively correlated with Pasteurellaceae, Enterobacteriaceae, and Gammaproteobacteria [38].

We also extended our prior work on carcinogen-induced rat colon tumors that examined miRNAs and the mRNA targets [17]. Mir-145 was among the most highly downregulated miRNAs in Pirc colon tumors, consistent with its proposed tumor suppressor role in human CRC, although mir- 145 was unexpectedly upregulated in carcinogen-induced colon tumors, for reasons that remain unclear. Previous studies prioritized SERPINE1 as a target of mir-143/145 in bladder cancer [39], as well as mir-34a in liver cancer, regulating KLF4 [40]. KLF4 is targeted by multiple miRNAs [40,41], including mir-34a, and in the Pirc model the mir-34a/ KIf4 axis was altered by SPI treatment. KLF4 is an important zinc-finger transcription factor involved in cell cycle regulation, somatic cell reprogramming, and tumorigenesis. Reduction of $K L F 4$ is well documented in rat $[41,42]$ and human colon tumors $[43,44]$, providing a potential avenue for next generation precision nutrition via SPI bioactives in the clinical setting [45].

Untargeted metabolomics corroborated several key findings from the microbiome and transcriptome studies, providing valuable insights and new mechanistic leads into the anticancer effects of SPI. Tumorassociated linoleate and its 15-LOX-1-dependent intermediates were lower in Pirc controls fed basal AIN diet, and they were normalized in adenomatous polyps after SPI treatment (Fig. 7a), consistent with the proposed anticancer mechanisms of these metabolites [25-27]. Several other intermediates associated with fatty acid metabolism also were detected in metabolomic analyses, such as 6-keto-prostaglandin $E_{1}$ (6-keto-PGE 1 ) and $\mathrm{PGE}_{2}$ ethanolamine; changes after SPI consumption were consistent with an overall shift towards anti-inflammatory, proapoptotic and tumor suppression pathways (Additional file 5). Thus, in SPI-fed rats, a decreased flux through pro-inflammatory leukotrienes and prostaglandins was paralleled by increased levels of 9-LOX, 15-LOX-1, prostacyclin, and cytochrome P450 (CYP) metabolites that are linked to anticancer outcomes [25-27].

One noteworthy observation was that enzymes associated with fatty acid metabolism (Additional file 5, blue font) were not among the main candidates prioritized by RNA-seq analyses (Additional file 4). We speculated that certain intermediates detected in rat tissues at 30 weeks (Fig. 1a) might derive directly from the SPI incorporated into the AIN basal diet, and this was confirmed via unbiased metabolomic analyses of the freeze-dried spinach. Thus, among the 700+ SPI analytes (Table 10 in Additional file 3), 
several corresponded to key intermediates detected in Pirc colon tumors and normal-looking tissues (Tables 9 and 10, Additional file 3, green font). These included linoleate, 13(S)-hydroperoxy-9Z,11Eoctadecadienoic acid (13-HPODE), and (9Z,11E)-13-oxooctadeca-9,11-dienoic acid (13-oxoODE), as well as L-glutamate and $\mathrm{N}$-acetylneuraminate. Interestingly, the 2-aceto-2-hydroxybutanoate that was increased in colon tumors from SPI-fed rats (Fig. 7b) was not detected in freeze-dried spinach, implicating beneficial butyrate-producing gut bacteria linked to increased a-diversity [46-48].

Although L-glutamate and $\mathrm{N}$-acetylneuraminate were detected via metabolomics in freeze-dried spinach, colon tumors at 30 weeks had reduced rather than increased levels of these intermediates following dietary SPI administration (Figs. 7c and 7d). Diminished L-glutamate levels in adenomatous polyps from SPI-fed rats would be synonymous with synthetic lethality [49], circumventing TCA cycle functions that are dependent on glutamine metabolism as a means of sustaining mitochondrial energetics. Lower levels of $\mathrm{N}$-acetylneuraminate in tumors from SPI-fed rats would implicate altered cell surface glycans that are critical for pathways in cancer, including immune evasion, resistance to apoptosis, and enhanced proliferation, metastasis and angiogenesis [50-52]. Notably, altered sialyation has been linked to activation of the inflammasome mediator elF2 [50], which in an APC-deficient background attenuates MYC-dependent apoptosis [53], unless circumvented by mechanisms that downregulate EIF2 - as observed for Eif2b2 in colon tumors from SPI-fed rats (Additional file 4, panel b, green font).

This investigation might be advanced in the future by further corroboration of mechanistic targets at the mRNA and protein level, by verification and quantification of key tumor tissue metabolites using refence standards and NMR-based methodologies, and by expanded metabolomic analyses that go beyond the hydrophilic analytes prioritized here, recognizing that chlorophylls and other lipophilic phytochemicals can exert anticancer effects in the colon [9-11, 54-60].

\section{Conclusions}

We demonstrated, for the first time, the marked anticancer efficacy of dietary spinach in the Apc-mutant Pirc model. After eliminating deregulated b-catenin as the primary mechanistic target, subsequent work identified significant reshaping of the gut microbiome in SPI-fed rats, along with changes in host transcriptomics and RNA-miRNA networks. Metabolomic analyses corroborated the predicted changes in linoleate and butanoate metabolism, TCA cycle, and pathways in cancer. Whereas butanoate metabolism was probably associated with increased a-diversity of the gut microbiome, multiple SPI-derived linoleate intermediates with reported anti-inflammatory and proapoptotic mechanisms were detected at increased levels in the colon tumors from rats treated with dietary SPI. Despite the presence of L-glutamate and $\mathrm{N}$ acetylneuraminate in freeze-dried spinach, these intermediates were reduced markedly in colon tumors from SPI-fed rats, consistent with anticancer outcomes of the associated metabolites. The latter findings warrant further investigation with respect to the potential for deregulated mitochondrial energetics leading to synthetic lethality [49], and altered cell surface glycans involved in immune evasion, oncogenic signaling networks and other pathways in cancer [50-53]. 


\section{Abbreviations}

Apc: Adenomatous polyposis coli; ATIMA: Agile Toolkit for Incisive Microbial Analyses; BrdU: bromodeoxyuridine, CMMR: Center for Metagenomic and Microbiome Research; CRC: colorectal cancer; COX: cyclooxygenases; Ctrl: control; CYP: cytochromes P450; DEGs: differentially expressed genes; DEmiRs: differentially expressed miRNAs; DHET: dihydroxyicosatrienoic acid; 8,9-DHET: 8,9-dihydroxy5Z,11Z,14Z-icosatrienoic acid; GO: Gene Ontology; GPx: glutathione peroxidase; GSEA: Gene Set Enrichment Analysis; H\&E: hematoxylin and eosin; HETE: hydroxyeicosatetraenoic acid; 13-HODE: (13S)hydroxyoctadecadienoic acid; 5-HPETE: 5-hydroperoxyeicosatetraenoic acid; KEGG: Kyoto Encyclopedia of Genes and Genomes; 6-keto-PGE1: 6-keto prostaglandin E1; 6-keto-PGF1a: 6-keto prostaglandin F1a; 9(S)HETE: (19S)-hydroxyeicosatetraenoic acid; 13-HPODE: 13S-hydroperoxy-9Z,11E-octadecadienoic acid; 9(S)-HPOT: (10E,12Z,15Z)-(9S)-9-hydroperoxyoctadeca-10,12,15-trienoic acid; LEfSe: LDA Effect Size; LTA $_{4}$ : leukotriene $\mathrm{A}_{4}$; miRNA: microRNA; 5-LOX: 5-lipoxygenase; 9-LOX: 9-lipoxygenase; 15-LOX-1: 15lipoxygenase-1; OTUs: Operational Taxonomic Units; 13-oxoODE: (9Z,11E)-13-oxooctadeca-9,11-dienoic acid; PARP: poly (ADP-ribose) polymerase; PCA: Principal Component Analysis; PCoA: Principal Coordinate Analysis; PGE2: prostaglandin E2; PICRUSt: Phylogenetic Investigation of Communities by Reconstruction of Unobserved States; Pirc: polyposis in rat colon; $\mathrm{PLA}_{2}$ : phospholipase $\mathrm{A}_{2}$; RNA-seq: RNA sequencing; RPKM: reads per kilo base per million mapped reads; RT-qPCR: reverse transcription quantitative polymerase chain reaction; SPI: spinach; STAMP: Statistical Analysis of Metagenomic Profiles; WT: wild type.

\section{Declarations}

\section{Acknowledgments}

We are grateful to Drs. Li Li, Praveen Rajendran, and Gavin Johnson (Center for Epigenetics \& Disease Prevention, Texas A\&M Health) for assistance with Pirc breeding and necropsies, Dr. Furkan Ertem (University of Pittsburgh School of Medicine) for training in murine endoscopy, and Dr. Nadim Ajami (Baylor College of Medicine) for consultation on microbiome analyses. Dr. Stephen Talcott (AgriLife Research, Texas A\&M University) kindly assisted with initial workup of spinach extraction procedures, and Drs. Praveen Rajendran, Sabeeta Kapoor, Yunus Demirhan, Nivedhitha Mohan and Jorge Tovar Perez (Texas A\&M Health) provided valuable discussion.

\section{Author contributions}

YSC performed preclinical, multi-omic, bioinformatic and molecular work and drafted the initial version of the manuscript; JL, KZ and DS assisted with bioinformatic and statistical analyses; YH guided library preparation for RNA-seq; WMD was responsible for animal breeding and genotyping, and assisted with necropsies and sample preparation; RM, AJ and KL oversaw metabolomic analyses; RHD participated in study design, overall project supervision, and final manuscript preparation. Authors read the manuscript and approved of its content. 


\section{Funding}

This work was supported by grants CA090890 and CA122959 from the National Cancer Institute, by the John S. Dunn Foundation, and by a Chancellor's Research Initiative.

\section{Availability of data and materials}

Datasets supporting this article will be made available with accession numbers in standard open access online repositories.

\section{Ethics approval and consent to participate}

No clinical trial was involved. Protocols in the rat received prior approval from the Institutional Animal Care and Use Committee at Texas A\&M University.

\section{Consent for publication}

Not applicable.

\section{Competing interests}

The authors declare that they have no competing interests.

\section{Author details}

${ }^{1}$ Center for Epigenetics \& Disease Prevention, Texas A\&M Health, Houston, TX, USA; ${ }^{2}$ Deparment of Chemical Engineering, College of Engineering, Texas A\&M University, College Station, TX, USA;

${ }^{3}$ Deparetment of Chemical and Biological Engineering, Tufts University, Medford, MA, USA; ${ }^{4}$ Department of Translational Medical Sciences, Texas A\&M College of Medicine, Houston, TX, USA.

\section{References}

1. Round JL, Mazmanian SK. The gut microbiota shapes intestinal immune responses during health and disease. Nat Rev Immunol. 2009;9:313-323.

2. Clemente JC, Ursell LK, Parfrey LW, Knight R. The impact of the gut microbiota on human health: an integrative view. 2012;148:1258-1270.

3. Ghaisas S, Maher J, Kanthasamy A. Gut microbiome in health and disease: linking the microbiomegut-brain axis and environmental factors in the pathogenesis of systemic and neurodegenerative diseases. Pharmacol Ther. 2016;158:52-62.

4. Tlaskalová-Hogenová H, Stepankova R, Kozakova H, Hudcovic T, Vannucci L, Tuckova L, Rossmann P, Nrncir T, Kverka M, Zakostelska Z, Klimesova K, Pribylova J, Bartova J, Sanchez D, Fundova P, Borovska D, Srutkova D, Zidek Z, Schwarzer M, Drastich P, Funda DP. The role of gut microbiota (commensal bacteria) and the mucosal barrier in the pathogenesis of inflammatory and 
autoimmune diseases and cancer: contribution of germ-free and gnotobiotic animal models of human diseases. Cell Mol Immunol. 2011:8:110-120.

5. Vannucci L, Stepankova R, Kozakova H, Fiserova A, Rossmann P, Tlaskalova-Hogenova. Colorectal carcinogenesis in germ-free and conventionally reared rats: different intestinal environments affect the systemic immunity. Int J Oncol. 2008;32:609-617.

6. Kelly CR, Khoruts A, Staley C, Sadowsky M, Abd M, Alani M, Bakow B, Curran P, McKenney J, Tisch A, Reinert SE, Machan JT, Brandt LJ. Effect of fecal microbiota transplantation on recurrence in multiple recurrent Clostridium difficile infection: a randomized trial. Ann Intern Med. 2016;165:609616.

7. Gupta S, Allen-Vercoe E, Petrof EO. Fecal microbiota transplantation: in perspective. Therap Adv Gastroenterol. 2016;9:229-239.

8. Nomura AMY, Wilkens LR, Murphy SP, Hankin JH, Henderson BE, Pike MC, Kolonel LN. Association of vegetable, fruit, and grain intakes with colorectal cancer: the Multiethnic Cohort Study. Am J Clin Nutr. 2008:88;730-737.

9. Gutierrez RMP, Velazquez EG, Carrera SPP. Spinach oleracea Linn considered as one of the most perfect foods: a pharmacological and phytochemical review. Mini Rev Med Chem. 2019;19:16661680.

10. Roberts JL, Moreau R. Functional properties of spinach (Spinacia oleracea) phytochemicals and bioactives. Food Funct. 2016:7;3337-3353.

11. Singh A, Singh P, Kumar B, Kumar S, Dev K, Maurya R. Detection of flavonoids from Spinacia oleracea leaves using HPLC-ESI-QTOF-MS/MS and UPLC-QqQLIT-MS/MS techniques. Nat Prod Res. 2019;33:2253-2256.

12. Ertem FU, Zhang W, Chang K, Dashwood WM, Rajendran P, Sun D, Abudayyeh A, Vilar E, Abdelrahim M, Dashwood RH. Oncogenic targets Mmp7, S100a9, Nppb and Aldh1a3 from transcriptome profiling of FAP and Pirc adenomas are downregulated in response to tumor suppression by Clotam. Int J Cancer. 2017;140:460-468.

13. Okonkwo A, Mitra J, Johnson GS, Li L, Dashwood WM, Hegde ML, Yue C, Dashwood RH, Rajendran P. Heterocyclic analogs of sulforaphane trigger DNA damage and impede DNA repair in colon cancer cells: interplay of HATs and HDACs. Mol Nutr Food Res. 2018;62:

14. Rajendran P, Johnson G, Li L, Chen YS, Dashwood WM, Nguyen N, Ulusan A, Ertem F, Zhang M, Li J, Sun D, Huang Y, Wang S, Leung HC, Lieberman D, Beaver L, Ho E, Bedford M, Chang K, Vilar E, Dashwood R. Acetylation of CCAR2 establishes a BET/BRD9 acetyl switch in response to combined deacetylase and bromodomain inhibition. Cancer Res. 2019;79:918-927.

15. Amos-Landgraf JM, Kwong LN, Kendziorski CM, Reichelderfer M, Torrealba J, Weichert J, Haag JD, Chen K-S, Waller JL, Gould MN, Dove WF. A target-selected Apc-mutant rat kindred enhances the modeling of familial human colon cancer. Proc Natl Acad Sci USA. 2007;104:4036-4041.

16. Irving AA, Yoshimi K, Hart M, Parker T, Clipson L, Ford MR, Kuramoto T, Dove WF, Amos-Landgraf JM. The utility of Apc-mutant rats in modeling human colon cancer. Dis Model Mech. 2014;7:1215-1225. 
17. Parasramka MA, Dashwood WM, Wang R, Abdelli A, Bailey GS, Williams DE, Ho E, Dashwood RH. MicroRNA profiling of carcinogen-induced rat colon tumors and the influence of dietary spinach. Mol Nutr Food Res. 2012;56:1259-1269.

18. Ertem F, Dashwood WM, Rajendran P, Raju G, Rashid A, Dashwood RH. Development of a murine colonoscopic polypectomy model (with videos). Gastrointest Endosc. 2016;83: 1272-1276.

19. Carmody RN,Gerber GK, Luevano JM, Gatti DM, Somes L, Svenson KL, Turnbaugh PJ. Diet dominates host genootype in shaping the murine gut microbiota. Cell Host Microbiome. 2015;17:72-84.

20. David LA, Maurice CF, Carmody RN, Gootenberg DB, Button JE, Wolfe BE, Ling AV, Devlin AS, Varma Y, Fischbach MA, Biddinger SB, Dutton RJ, Turnbaugh PJ. Diet rapidly and reproducibly alters the human gut microbiome. Nature. 2014;505:559-563.

21. Son JS, Khair S, Pettet $3^{\text {rd }}$ DW, Quyang N, Tian X, Zhang Y, Zhu W, Mackenzie GG, Robertson CE, Ir D, Frank DN, Rigas B, Li E. Altered interactions between the gut microbiome and colonic mucosa precede polyposis in ApcMin/+ mice. PLoS One. 2015;10:

22. Dai Z, Coker OO, Nakatsu G, Wu WKK, Zhao L, Chen Z, Chan FKL, Kristiansen K, Sung JJY, Wong SH, Yu J. Multi-cohort analysis of colorectal cancer metagenome identified altered bacteria across populations and universal bacterial markers. Microbiome. 2018;6:70.

23. Fritz JV, Desai MS, Shah P, Schneider JG, Wilmes P. From meta-omics to causality: experimental models for human microbiome research. Microbiome. 2013;1:14.

24. Venkataraman A, Sieber JR, Schmidt AW, Waldron C, Theis KR, Schmidt TM. Variable responses of human microbiomes to dietary supplementation with resistant starch. 2016;4:33.

25. Vangaveti VN, Jansen H, Kennedy RL, Malabu US. Hydroxyoctadecadienoic acids: oxidized derivatives of linoleic acid and their role in inflammation associated with metabolomic syndrome and cancer. Eur J Pharmacol. 2016;785:70-76.

26. Lee SI, Zuo X, Shureiqi I. 15-Lipoxygenase-1 as a tumor suppressor gene in colon cancer: is the verdict in? Cancer Metastasis Rev. 2011;30:481-491.

27. Liu F, Zuo X, Liu Y, Deguchi Y, Moussalli MJ, Chen W, Yang P, Wei B, Tan L, Lorenzi PL, Gao S, Jaoude JC, Mehdizadeh A, Valentin LA, Wei D, Shureiqi I. Suppression of membranous LRP5 recycling, Wnt/b-catenin signaling, and colon carcinogenesis by 15-LOX-1 peroxidation of linoleic acid in PIP. Cell Rep. 2020;32:108049.

28. Tsilimigras MCB, Fodor A, Jobin C. Carcinogenesis and therapeutics: the microbiota perspective. Nat Microbiol. 2017;2:17008.

29. Ahn J, Sinha R, Pei Z, Dominianni C, Wu J, Shi J, Goedert JJ, Hayes RB, Yang L. Human gut microbiome and risk for colorectal cancer. J Natl Cancer Inst. 2013;105:1907-1911.

30. Salonen A, Lahti L, Salojarvi J, Holtrop G, Korpela K, Duncan SH, Date P, Farquharson F, Johnstone AM, Lobley GE, Louis P, Flint HJ, de Vos WM. Impact of diet and individual variation on intestinal microbiota composition and fermentation products in obese men. ISME J. 2014;8:2218-2230. 
31. Rey FE, Gonzalez MD, Cheng J, Wu M, Ahern PP, Gordon JI. Metabolic niche of a prominent sulfatereducing human gut bacterium. Proc Natl Acad Sci USA. 2013;110:13582-13587.

32. Jakobsson HE, Rodriguez-Pineiro AM, Schutte A, Ermund A, Boysen P, Bemark M, Sommer F, Backhed F, Hansson GC, Johansson MEV. The composition of the gut microbiota shapes the colon mucus barrier. EMBO Rep. 2015;16:164-77.

33. Ghosh AK, Vaughan DE. PAl-1 in tissue fibrosis. J Cell Physiol. 2012;227:493-507.

34. Sakakibara T, Hibi K, Koike M, Fujiwara M, Kodera Y, Ito K, Nakao A. Plasminogen activator inhibitor-1 as a potential marker for the malignancy of colorectal cancer. Br J Cancer. 2005;93:799-803.

35. Ganesh S, Sier CF, Griffioen G, Vloedgraven HJ, de Boer A, Welvaart K, van de Velde CJ, van Krieken $\mathrm{JH}$, Verheijen JH, Lamers CB, Verspaget HW. Prognostic relevance of plasminogen activators and inhibitors in colorectal cancer. Cancer Res. 1994;54:4065-4071.

36. Flemer B, Lynch DB, Brown JM, Jeffrey IB, Ryan FJ, Claesson MJ, O'Riordain M, Shanahan F, O'Toole PW. Tumour-associated and non-tumour-associated microbiota in colorectal cancer. 2017;66:633643.

37. Wang R, Dashwood WM, Nian H, Lohr, Fischer KA, Tsuchiya N, Nakagama H, Ashktorab H, Dashwood $\mathrm{RH}$. NADPH oxidase overexpression in human colon cancers and rat colon tumors induced by 2amino-1-methyl-6-phenylimidazo[4,5- $b$ ]pyridine (PhIP). Int J Cancer. 2011;128:2581-2590.

38. Haberman Y, Tickle TL, Dexheimer PJ, Kim MO, Tang D, Karns R, Baldassano RN, Noe JD, Rosh J, Markowitz J, Heyman MB, Griffiths AM, Crandall WV, Mack DR, Baker SS, Huttenhower C, Keljo DJ, Hyams JS, Kugathasan S, Walters TD, Aronow B, Xavier RJ, Gevers D, Denson LA. Pediatric Crohn disease patients exhibit specific ileal transcriptome and microbiome signature. J Clin Invest. 2014;124:3617-3633.

39. Villadsen SB, Bramsen JB, Ostenfeld MS, Wiklund ED, Fristrup N, Gao S, Hansen TB, Jensen TI, Borre $M$, Orntoft TF, Dyrskjot L, Kjems J. The miR-143/-145 cluster regulates plasminogen activator inhibitor-1 in bladder cancer. Br J Cancer. 2012;106:366-374.

40. Chen Q, Li L, Tu Y, Zheng LL, Liu W, Zuo XY, He YM, Zhang SY, Zhu W, Cao JP, Cui FM, Hou J. MiR-34a regulates apoptosis in liver cells by targeting the KLF4 gene. Cell Mol Biol Lett. 2014;19:52-64.

41. Parasramka MA, Dashwood WM, Wang R, Saeed HH, Williams DE, Ho E, Dashwood RH. A role for low-abundance miRNAs in colon cancer: the miR-206/Krüppel-like factor 4 (KLF4) axis. Clin Epigenetics. 2012;4:

42. Chen Y-S, Wang R, Dashwood WM, Lohr CV, Williams DE, Ho E, Mertens-Talcott S, Dashwood RH. A miRNA signature for an environmental heterocyclic amine defined by a multi-organ carcinogenicity bioassay in the rat. Arch Toxicol. 2017;91:3415-3425.

43. Xu J, Lu B, Xu F, Gu H, Fang Y, Huang Q, Lai M. Dynamic down-regulation of Krüppel-like factor 4 in colorectal adenoma-carcinoma sequence. J Cancer Res Clin Oncol. 2008;134:891-898.

44. Patel NV, Ghaleb AM, Nandan MO, Yang VW. Expression of the tumor suppressor Krüppel-like factor 4 as a prognostic predictor for colon cancer. Cancer Epidemiol Biomarkers Prev. 2010;19:2631-2638. 
45. Chakraborty C, Sharma AR, Sharma G, Doss CGP, Lee SS. Therapeutic miRNA and siRNA: moving from bench to clinic as next generation medicine. Mol Ther Nucleic Acids. 2017;8:132-143.

46. Yi Y, Shen L, Shi W, Xia F, Zhang H, Wang Y, Zhang J, Wang Y, Sun X, Zhang Z, Zou W, Yang W, Zhang L, Zhu J, Goel A, Ma Y, Zhang Z. Gut microbiome components predict response to neoadjuvant chemoradiotherapy in locally advanced rectal cancer patients: a prospective, longitudinal study. Clin Cancer Res. 2020; DOI: 1158/1078-0432.CCR-20-3445.

47. Ocvirk S, Wilson AS, Posma JM, Li JV, Koller KR, Day GM, Flanagan CA, Otto JE, Sacco PE, Sacco FD, Sapp FR, Wilson AS, Newton K, Brouard F, DeLany JP, Behnning M, Appolonia CN, Soni D, Bhatti F, Methe B, Fitch A, Morris A, Gaskins HR, Kinross J, Nicholson JK, Thomas TK, O'Keefe SJD. A prospective cohort analysis of gut microbial co-metabolism in Alaska Native and rural African people at high and low risk of colorectal cancer. Am J Clin Nutr. 2020;111:406-419.

48. Chen D, Jin D, Huang S, Wu J, Xu M, Liu T, Dong W, Liu X, Wang S, Zhong W, Liu Y, Jiang R, Piao M, Wang B, Cao H. Clostridium butyricum, a butyrate-producing probiotic, inhibits intestinal tumor development through modulating Wnt signaling and gut microbiota. Cancer Lett. 2020;469:456-467.

49. Yang C, Ko B, Hensley CT, Jiang L, Wasti AT, Kim J, Sudderth J, Calvaruso MA, Lumata L, Mitsche M, Rutter J, Merritt ME, DeBerardinis RJ. Glutamine oxidation maintains the TCA cycle and cell survival during impaired mitochondrial pyruvate transport. Mol Cell. 2014;56:414-424.

50. Giron LB, Tanes CE, Schleimann MH, Engen PA, Mattei LM, Anzurez A, Damra M, Zhang H, Bittinger K, Bushman F, Kossenkov A, Denton PW, Tateno H, Keshavarzian A, Landay AL, Abdel-Mohsen M. Sialylation and fucosylation modulate inflammasome-activating elF2 signaling and microbial translocation during HIV infection. Mucosal Immunol. 2020;13:753-766.

51. Kotsias M, Blanas A, van Vliet SJ, Pirro M, Spencer DIR, Kozak RP. Method comparison for N-glycan profiling: towards the standardization of glycolanalytical technologies for cell line analysis. PloS One 2019;14:e0223270.

52. Zhou X, Yang G, Guan F. Biological functions and analytical strategies of sialic acids in tumor. Cells. 2020;9:273.

53. Schmidt S, Gay D, Uthe FW, Denk S, Paauwe M, Matthes N, Diefenbacher ME, Bryson S, Warrander FC, Erhard F, Ade CP, Baluapuri A, Walz S, Jackstadt R, Ford C, Vlachogiannis G, Valeri N, Otto C, SchüleinVölk C, Maurus K, Schmitz W, Knight JRP, Wolf E, Strathdee D, Schulze A, Germer CT, Rosenwald A, Sansom OJ, Eilers M, Wiegering A. A MYC-GCN2-elF2a negative feedback loop limits protein synthesis to prevent MYC-dependent apoptosis in colorectal cancer. Nat Cell Biol. 2019;21:14131424.

54. Dashwood RH, Breinholt V, Bailey GS. Chemopreventive properties of chlorophyllin: inhibition of aflatoxin B1 (AFB1)-DNA binding in vivo and anti-mutagenic activity against AFB1 and two heterocyclic amines in the Salmonella mutagenicity assay. Carcinogenesis. 1991;12:939-942.

55. Dashwood R, Guo D. Inhibition of 2-amino-3-methylimidazo[4,5- $f$ qquinoline (IQ)-DNA binding by chlorophyllin: studies of enzyme inhibition and molecular complex formation. Carcinogenesis. 1992;13:1121-1126. 
56. Guo D, Horio DT, Grove JS, Dashwood RH. Inhibition by chlorophyllin of 2-amino-3methylimidazo[4,5-f]quinoline-induced tumorigenesis in the male F344 rat. Cancer Lett. 1995;95:161165.

57. Diaz GD, Li Q, Dashwood RH. Caspase-8 and apoptosis-inducing factor mediate a cytochrome $c$ independent pathway of apoptosis in human colon cancer cells induced by the dietary phytochemical chlorophyllin. Cancer Res. 2003;63:1254-1261.

58. Simonich MT, Egner PA, Roebuck BD, Orner GA, Jubert C, Pereira C, Groopman JD, Kensler TW, Dashwood RH, Williams DE, Bailey GS. Natural chlorophyll inhibits aflatoxin B1-induced multi-organ carcinogenesis in the rat. Carcinogenesis. 2007;28:1294-1302.

59. Jubert C, Mata J, Bench G, Dashwood R, Pereira C, Tracewell W, Turteltaub K, Williams D, Bailey G. Effects of chlorophyll and chlorophyllin on low-dose aflatoxin B1 pharmacokinetics in human volunteers. Cancer Prev Res. 2009;2:1015-1022.

60. Egner PA, Wang JB, Zhu YR, Zhang BC, Wu Y, Zhang QN, Qian GS, Kuang SY, Gange SJ, Jacobson LP, Helzlsouer KJ, Bailey GS, Groopman JD, Kensler TW. Chlorophyllin intervention reduces aflatoxinDNA adducts in individuals at high risk for liver cancer. Proc Natl Acad Sci U S A. 2001;98:1460114606.

61. Wang R, Dashwood WM, Lohr CV, Fischer KA, Nakagama H, Williams DE, Dashwood RH. beta-Catenin is strongly elevated in rat colonic epithelium following short-term intermittent treatment with 2amino-1-methyl-6-phenylimidazo[4,5-b]pyridine (PhIP) and a high-fat diet. Cancer Sci. 2008;99:17541759.

62. Myzak, MC, Karplus PA, Chung F-L, Dashwood RH. A novel mechanism of chemoprotection by sulforaphane: inhibition of histone deacetylase. Cancer Res. 2004;64:5767-5774.

63. Rajendran P, Dashwood WM, Li L, Kang Y, Kim E, Johnson G, Fischer KA, Lohr CV, Williams DE, Ho E, Yamamoto M, Lieberman DA, Dashwood RH. Nrf2 status affects tumor growth, HDAC3 gene promoter associations, and the response to sulforaphane in the colon. Clin Epigenetics. 2015;7:

64. Rajendran P, Delage B, Dashwood RH, Yu T-W, Wuth B, Williams DE, Ho E, Dashwood RH. Histone deacetylase turnover and recovery in sulforaphane-treated colon cancer cells: competing actions of 14-3-3 and Pin1 in HDAC3/SMRT corepressor complex dissociation/reassembly. Mol Cancer. 2011;10:

65. Larsen CA, Dashwood RH. (-)-Epigallocatechin-3-gallate inhibits Met signaling, proliferation, and invasiveness in human colon cancer cells. Arch Biochem Biophys. 2010;501:52-57.

66. Segata N, Izard J, Waldron L, Gevers D, Miropolsky L, Garrett WS, Huttenhower C. Metagenomic biomarker discovery and explanation. Genome Biol. 2011;12:

67. Langille MGI, Zaneveld J, Caporaso JG, McDonald D, Knights D, Reyes JA, Clemente JC, Burkepile DE, Vega Thurber RL, Knight R, Beiko RG, Huttenhower C. Predictive functional profiling of microbial communities using 16S rRNA marker gene sequences. Nat Biotechnol. 2013;31:814-821.

68. Parks DH, Tyson GW, Hugenholtz P, Beiko RG. STAMP: statistical analysis of taxonomic and functional profiles. 2014;30:3123-3124. 
69. Xia J, Psychogios N, Young N, Wishart DS. MetaboAnalyst: a web server for metabolomic data analysis and interpretation. Nucleic Acids Res. 2009;37:W652-W660.

70. Xia J, Wishart DS. Web-based inference of biological patterns, functions and pathways from metabolomic data using MetaboAnalyst. Nat Protoc. 2011;6:743-760.

71. Cheng K, Ning Z, Zhang X, Li L, Liao B, Mayne J, Stintzi A, Figeys D. MetaLab: an automated pipeline for metaproteomic data analysis. Microbiome. 2017;5:157.

72. Li L, Ning Z, Zhang X, Mayne J, Cheng K, Stintzi A, Figeys D. RapidAIM: a culture- and metaproteomics-based rapid assay of individual microbiome responses to drugs. Microbiome. 2020;8:33.

73. Li S, Park Y, Duraisingham S, Strobel FH, Khan N, Soltow QA, Jones DP, Pulendran B. Predicting network activity from high throughput metabolomics. PloS Comput Biol. 2013;9:e1003123.

74. Shannon P, Markiel A, Ozier O, Baliga NS, Wang JT, Ramage D, Amin N, Schwikowski B, Ideker T. Cytoscape: a software environment for integrated models of biomolecular interaction networks. Genome Res. 2003;13:2498-2503.

75. Langmead B, Salzberg SL. Fast gapped-read alignment with Bowtie 2. Nat Methods. 2012;9:357359.

76. Anders S, PyI PT, Huber W. HTSeq - A Python framework to work with high-throughput sequencing data. Bioinformatics. 2015;31:166-169.

77. Martin M. Cutadapt removes adapter sequences from high-throughput sequencing reads. EMBnet $j$. 2011;17:

78. Agarwal V, Bell GW, Nam J-W, Bartel DP. Predicting effective microRNA target sites in mammalian mRNAs. 2015;4:e05005.

79. Wang R, Lohr, Fischer KA, Dashwood WM, Greenwood JA, Ho E, Williams DE, Ashktorab H, Dashwood MR, Dashwood RH. Epigenetic inactivation of endothelin-2 and endothelin-3 in colon cancer. Int $J$ Cancer. 2013;132:1004-1012.

80. Nian H, Bisson WH, Dashwood RH, Pinto JT, Dashwood RH. a-Keto acid metabolites of organoselenium compounds inhibit histone deacetylase activity in human colon cancer cells. Carcinogenesis. 2009;30:1416-1423.

81. Blum CA, Xu M, Orner GA, Fong TA, Bailey GS, Stoner GD, Horio DT, Dashwood RH. beta-Catenin mutation in rat colon tumors initiated by 1,2-dimethylhydrazine and 2-amino-3-methylimidazo[4,5$f$ qquinoline, and the effect of post-initiation treatment with chlorophyllin and indole-3-carbinol. Carcinogenesis. 2001;22:315-320.

82. Dashwood RH, Xu M, Hernaez JF, Hasaniya N, Youn K, Razzuk A. Cancer chemopreventive mechanisms of tea against heterocyclic amine mutagens from cooked meat. Proc Exp Biol Med 1999;220:239-243.

83. Dashwood RH, Uyetake L, Fong AT, Hendricks JD, Bailey GS. In vivo disposition of the natural anticarcinogen indole-3-carbinol after po administration. Food Chem Toxicol. 1989;27:385-392. 
84. Li J, Zhao S, Lee M, Yin Y, Li J, Zhou Y, Ballester LY, Esquenazi Y, Dashwood RH, Davies PJA, Parsons DW, Li XN, Huang Y, Sun D. Reliable tumor detection by whole-genome methylation sequencing of cell-free DNA in cerebrospinal fluid of pediatric medulloblastoma. Sci Adv. 2020;6:eabb5427.

\section{Figures}

a

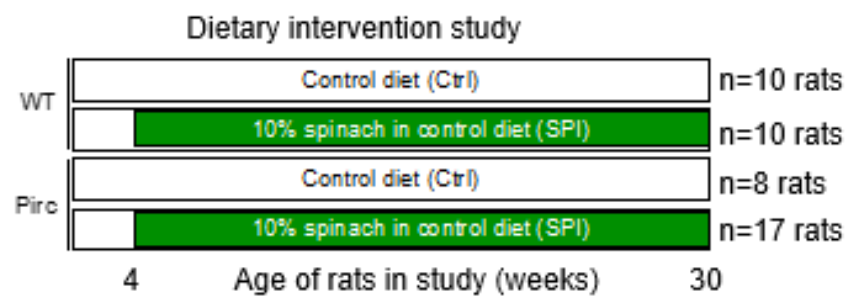

C

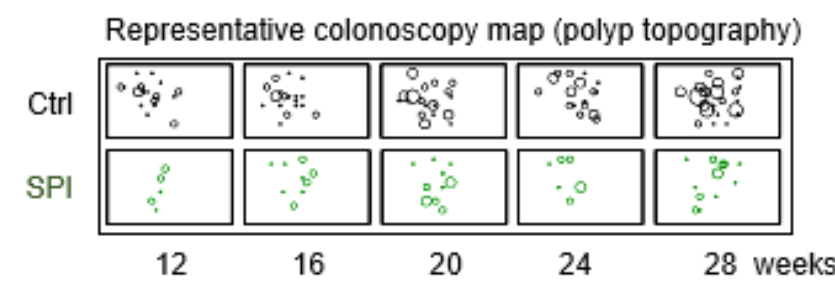

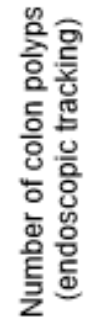

e
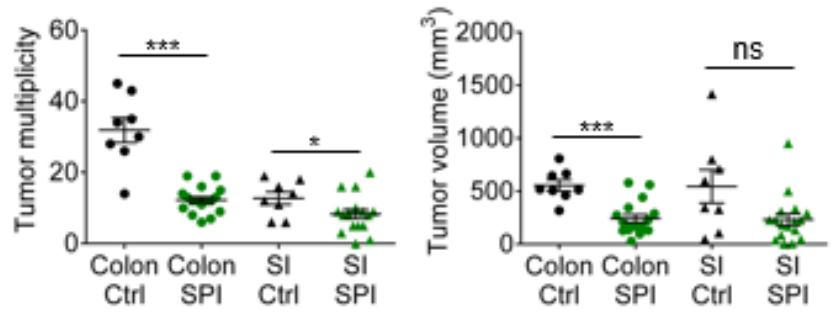

b

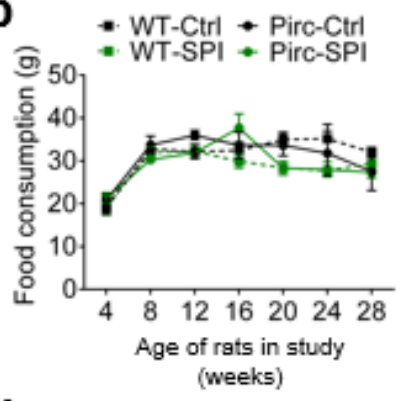

d

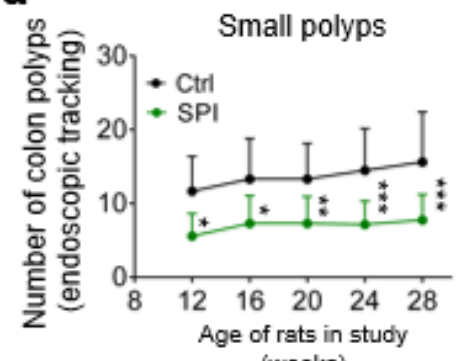

(weeks)
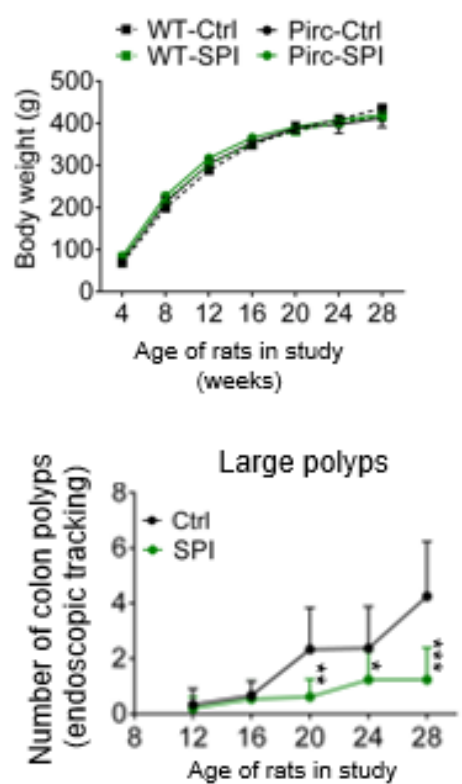

(weeks)
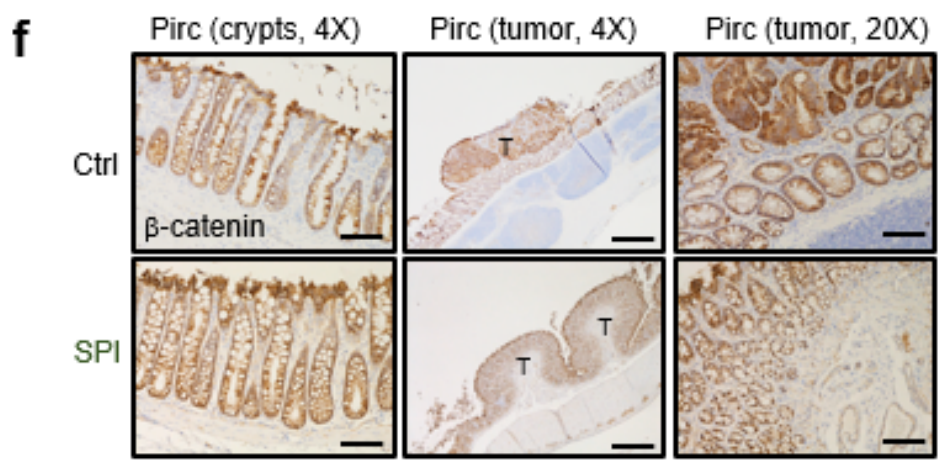

Figure 1

Dietary spinach suppresses tumor development in an Apc-mutant rat model. (a) Study design in Pirc and WT rats; $\mathrm{n}=10$ for WT-Ctrl (wild type rats given control/basal AIN diet) and WT-SPI (WT rats given AIN diet containing $10 \%$ freeze-dried spinach), $n=8$ for Pirc-Ctrl, and $n=17$ for Pirc-SPI. (b) Food consumption and body wt. gain. (c) Monthly endoscopic tracking of colon polyps in the Pirc model. Topography images reflect progressive changes observed in polyp size and location, whereas the bar graph represents average multiplicity assessed via endoscopy. (d) Number of small (size grade 1-3) and large polyps (size grade 4-5) by monthly endoscopy, based on a reported grading system [18]. (e) Final tumor outcomes for colon and small intestine (SI) polyps at the 30-wk necropsy. (f) Immunohistochemistry staining of $\beta$ - 
catenin in Pirc rats given Ctrl or SPI diets. Scale bar, $100 \mu \mathrm{m}$ for $20 \mathrm{X}$ and $500 \mu \mathrm{m}$ for 4X magnification. Numerical data are presented as mean \pm SEM; ${ }^{*} p<0.05,{ }^{* \star} p<0.01,{ }^{\star *} p<0.001$, ns statistically nonsignificant.
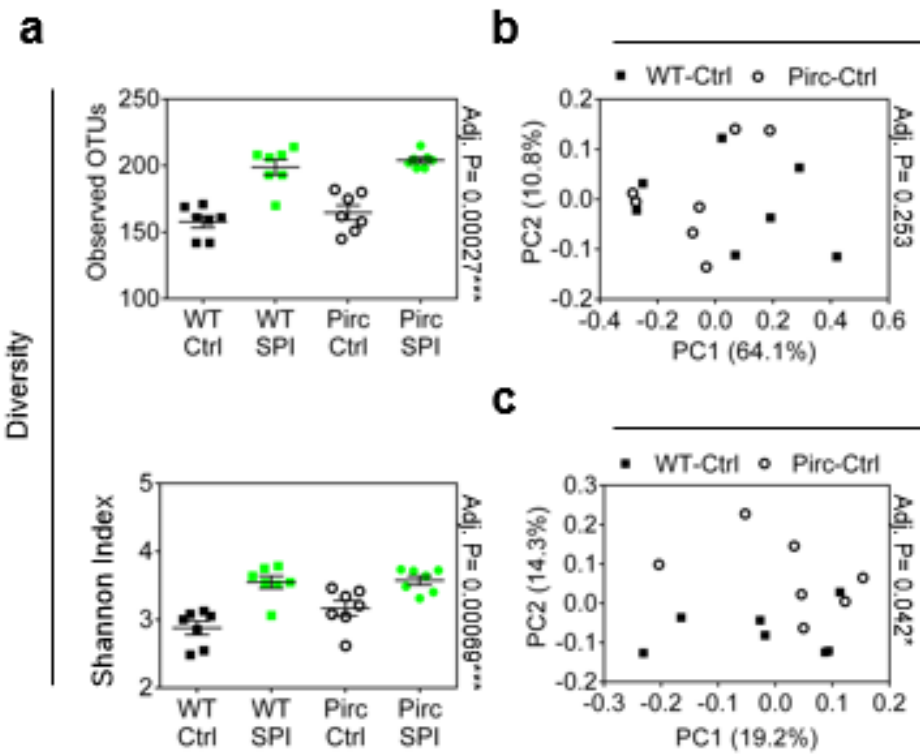

Weighted UniFrac PCoA

C
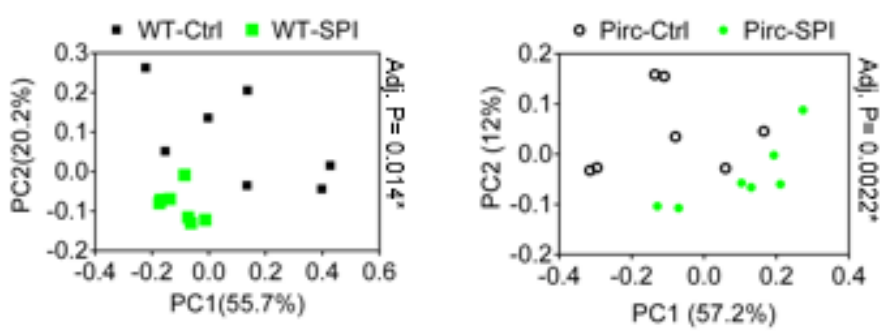

Unweighted UniFrac PCoA
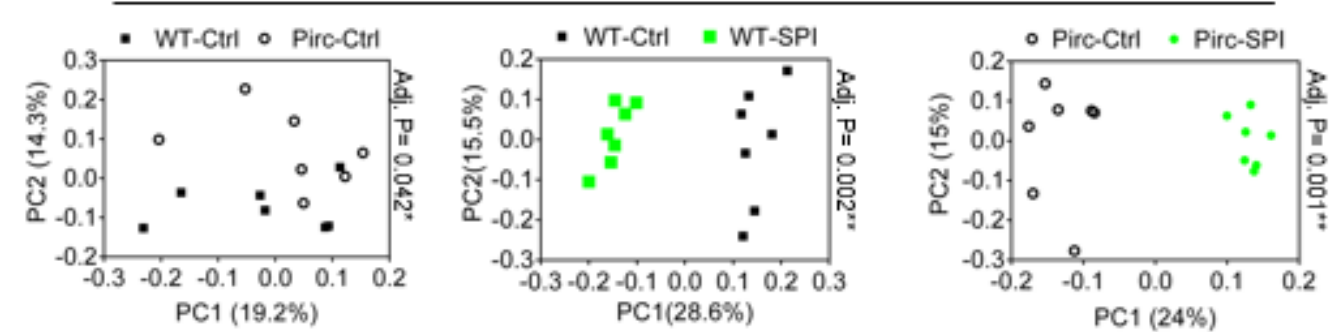

d

Phylum
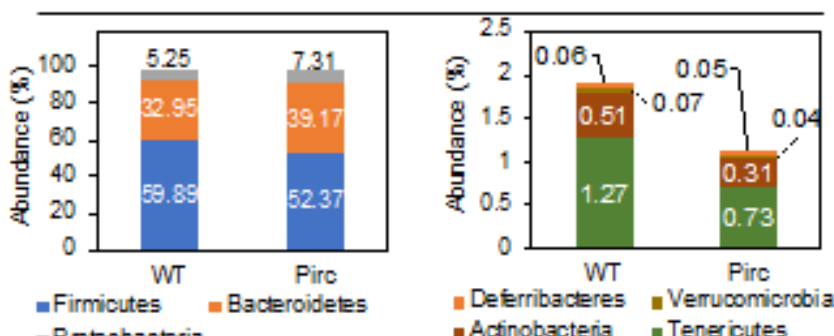

e

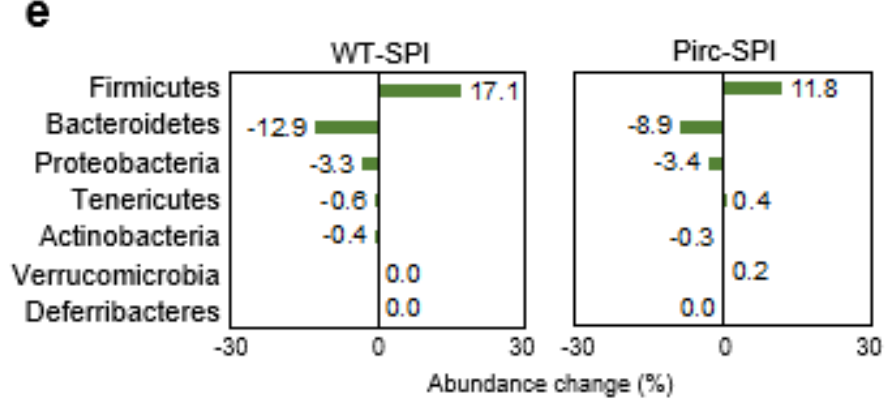

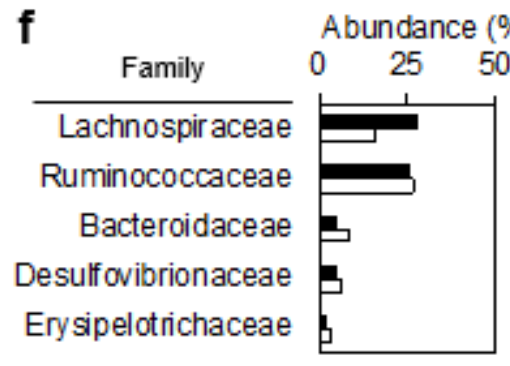

-WT-Ctrl aPirc-Ctrl
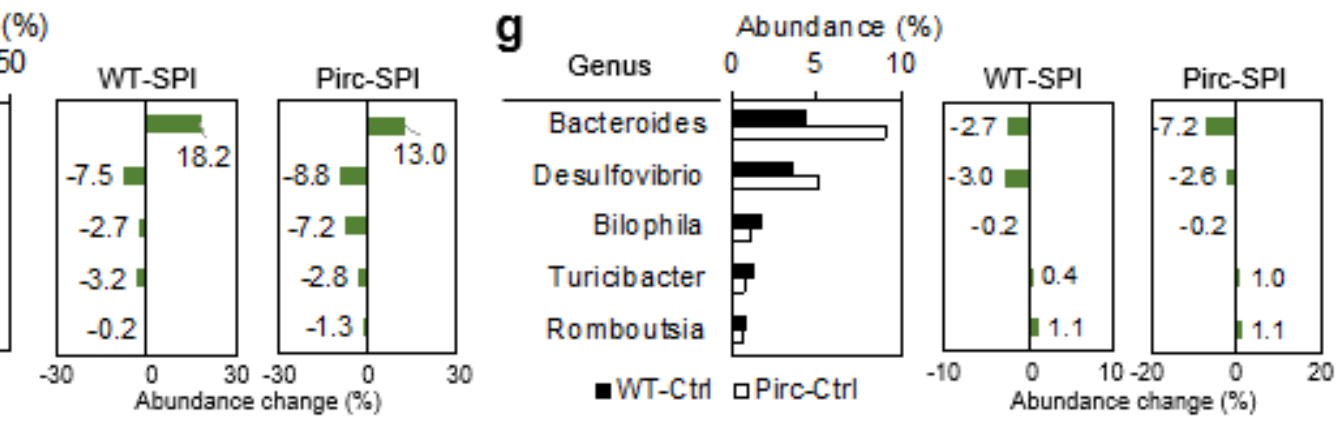

Figure 2

Microbial composition in relation to host genotype and dietary intervention. (a) a-diversity measured by observed OTUs and Shannon index; $\mathrm{n}=7$ replicates for $16 \mathrm{~S}$ sequencing in each group. (b) Weighted and (c) unweighted UniFrac principal coordinate analysis (PCoA) plots displaying $\beta$-diversity. (d) Taxonomic composition in Pirc and WT rats at the phylum level. (e) Relative abundance changes at the phylum level after SPI intake. (f) Left, top five-most abundant family members. Right, abundance changes by SPI in Pirc and WT rats. (g) Left, top five most abundant genera. Right, abundance changes by SPI in Pirc and WT rats. Adjusted $p$ values were calculated by Kruskal-Wallis test. 

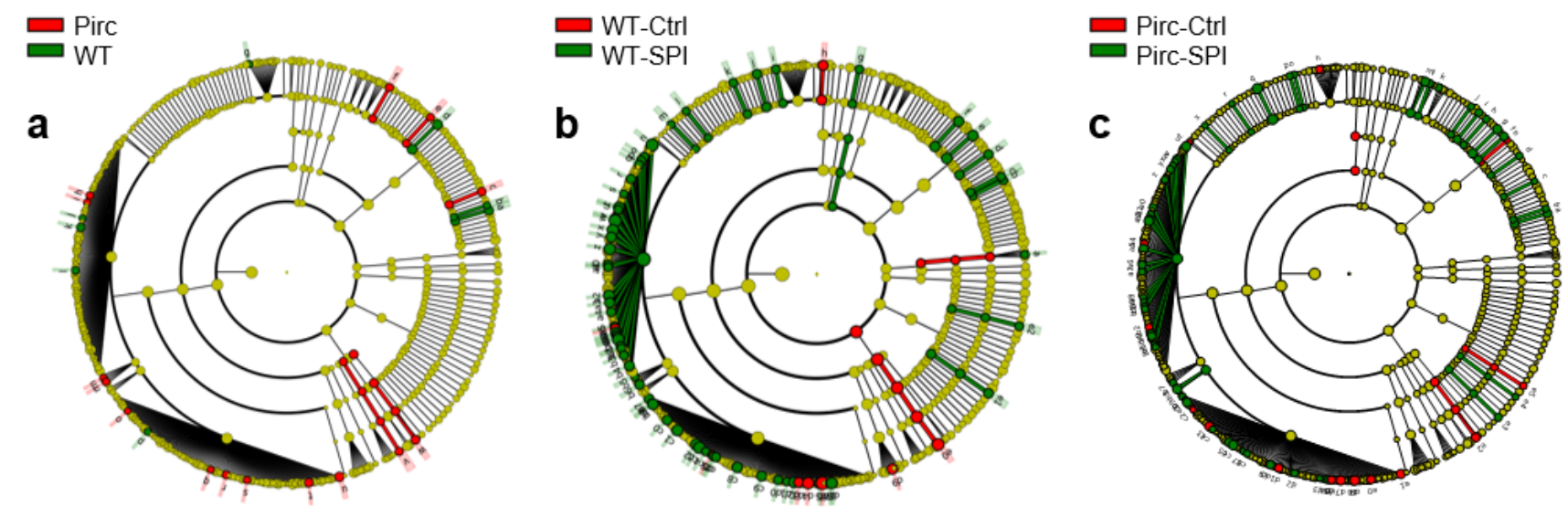

LDA score $(\log 10)$

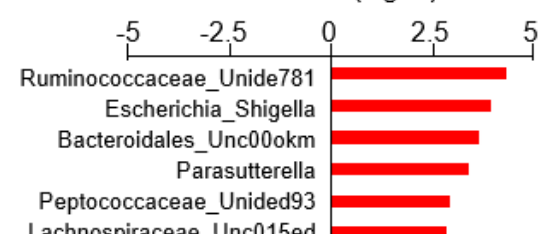

LDA score $(\log 10)$

LDA score $(\log 10)$
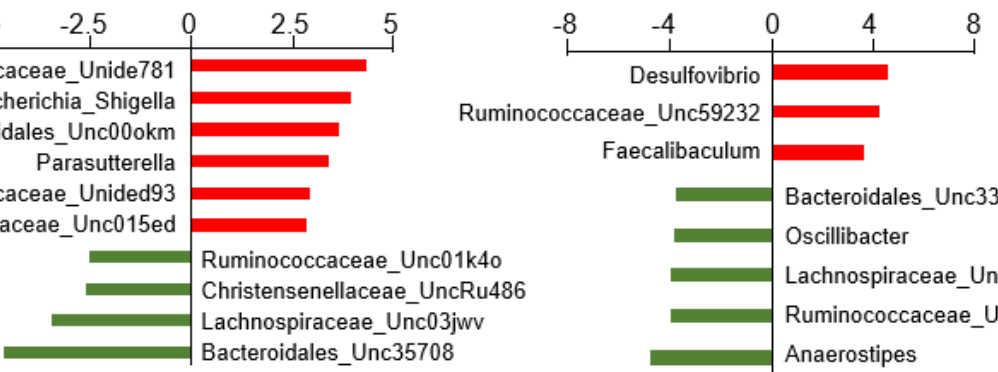

Faecalibaculum
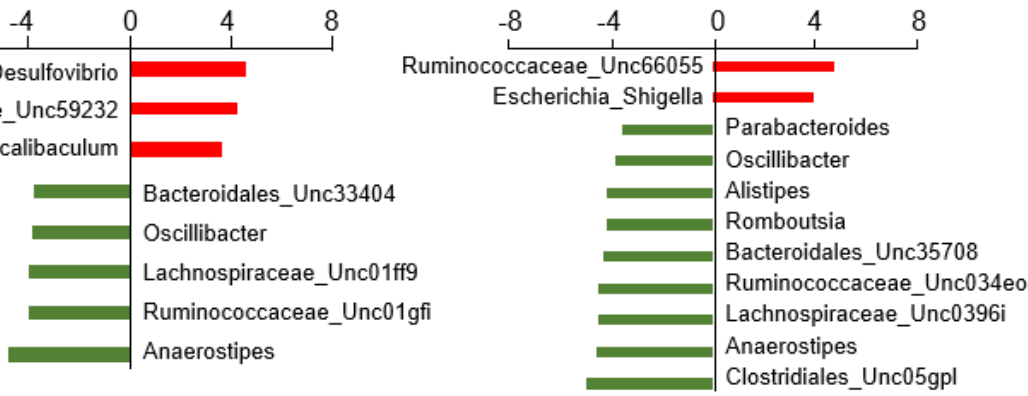

d

LEfSe

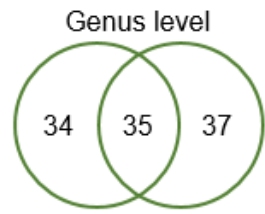

WT-SPI Pirc-SPI

e PICRUSt

Metagenome prediction

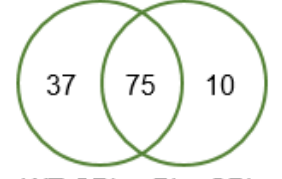

WT-SPI Pirc-SPI

g

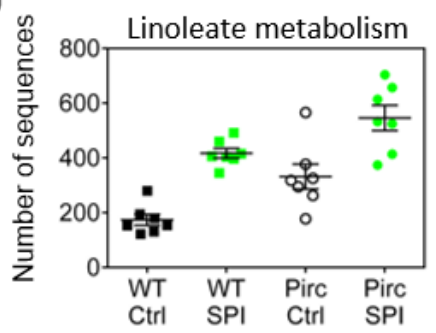

Porphyrin and chlorophyll metabolism

Butanoate metabolism

Protein kinases

Diff between Rel. Freq. (\%)

f

$\square$ WT-SPI $\square$ Pirc-SPI

$\square$ WT-SPI $\square$ Pirc-SPI
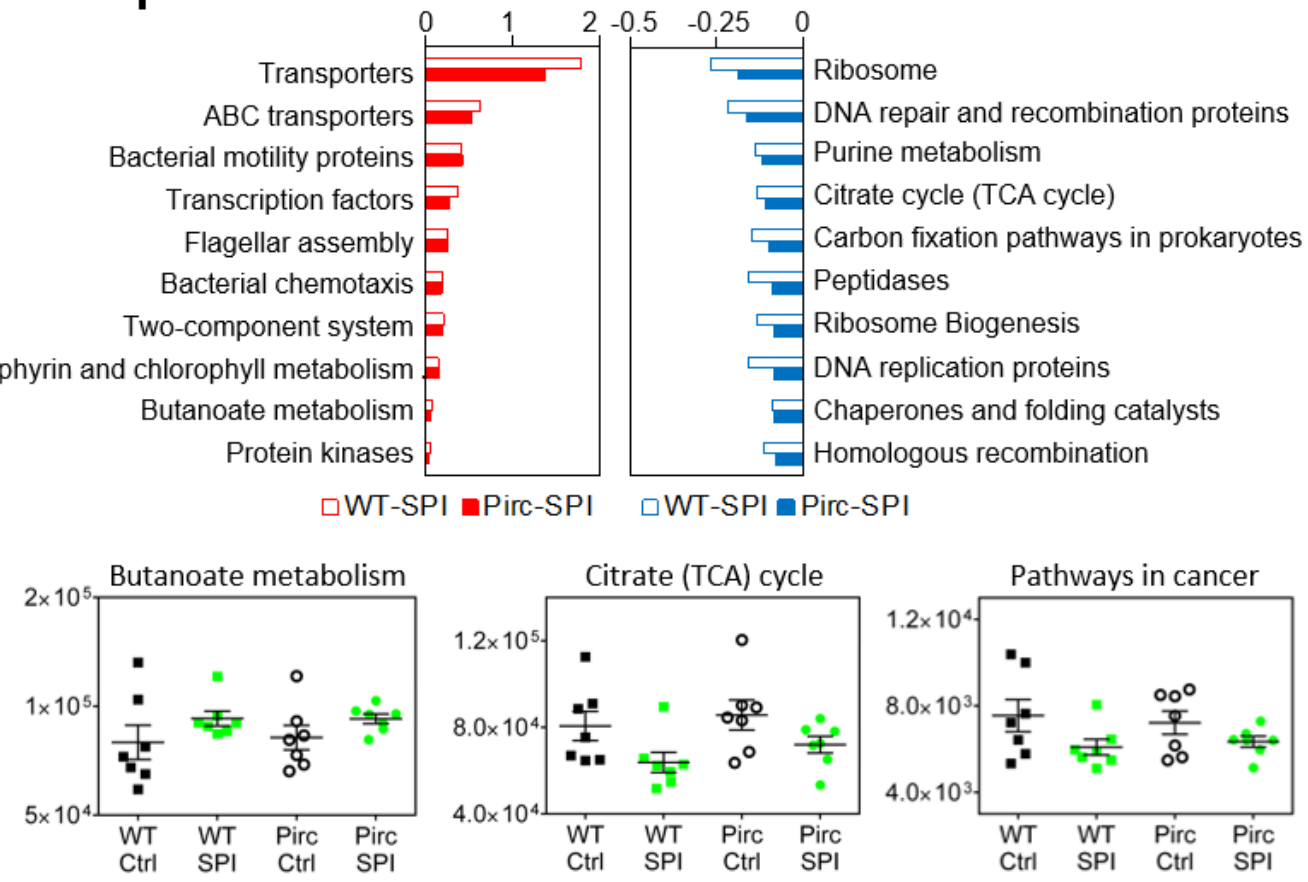

\section{Figure 3}

Dietary spinach reshapes the microbiome and its predicted metagenome. Cladograms and LDA scores for enriched clades in two-group comparisons of LEfSe data: (a) WT vs. Pirc (no SPI treatment); (b) WT $\pm S P I$; (c) Pirc $\pm S P I$. Cladograms illustrate all significant changes in taxonomy, whereas the bar graphs below show representative enriched bacteria taxonomy. Venn diagrams of (d) LEfSe data for enriched clades at the genus level and (e) metagenome prediction from PICRUSt in Pirc and WT rats after SPI 
treatment. (f) Prediction of top ten functional metagenomes in Pirc and WT rats given SPI. (g) Linoleate $(p=2.19 \mathrm{e}-5)$ and butanoate metabolism $(p=1.44 \mathrm{e}-9)$ were increased significantly, whereas TCA cycle $(p=9.86 \mathrm{e}-4)$ and Pathways in Cancer $(p=2.85 \mathrm{e}-3)$ were decreased by SPI treatment in Pirc and WT rats. Statistical analyses were performed in STAMP with ANOVA. Tables 2-5 in Additional file 3 provide complete LDA results, unclassified 16S rRNA seq data, and detailed PICRUSt information.

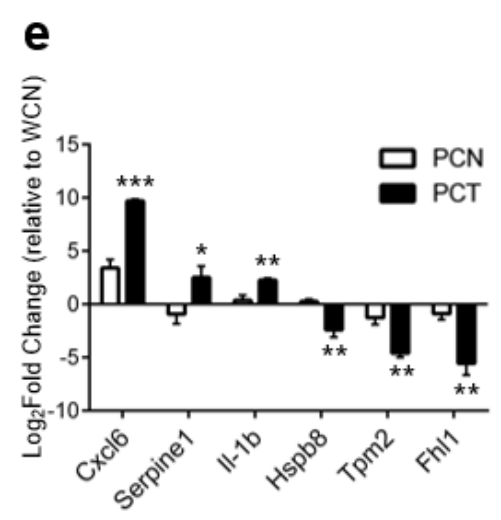

g
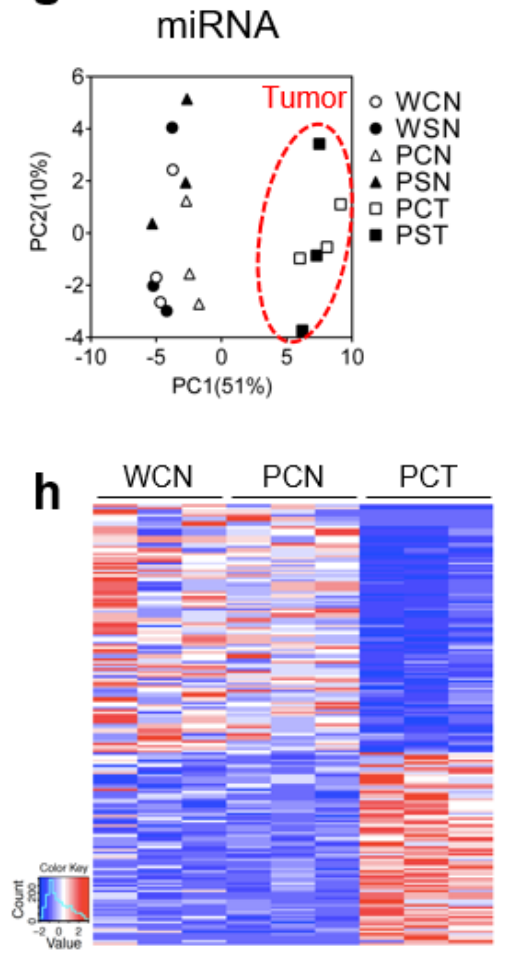

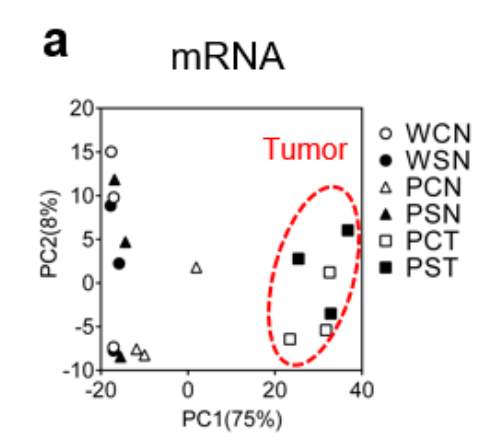

b
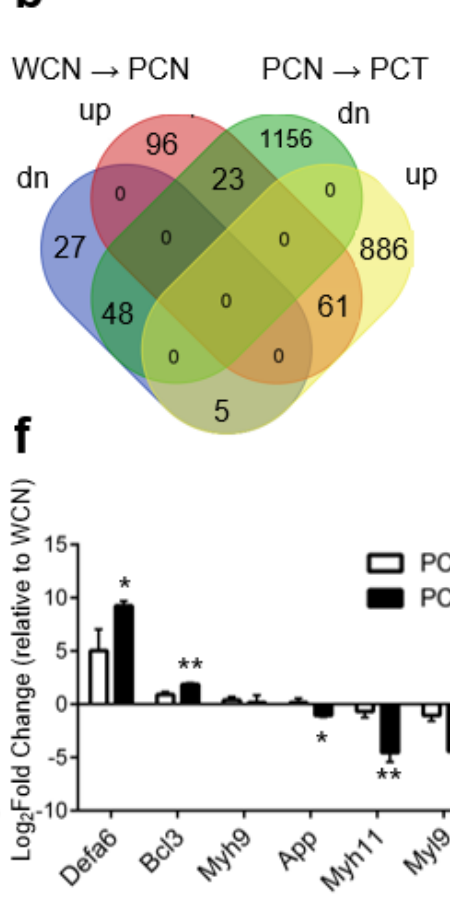

i

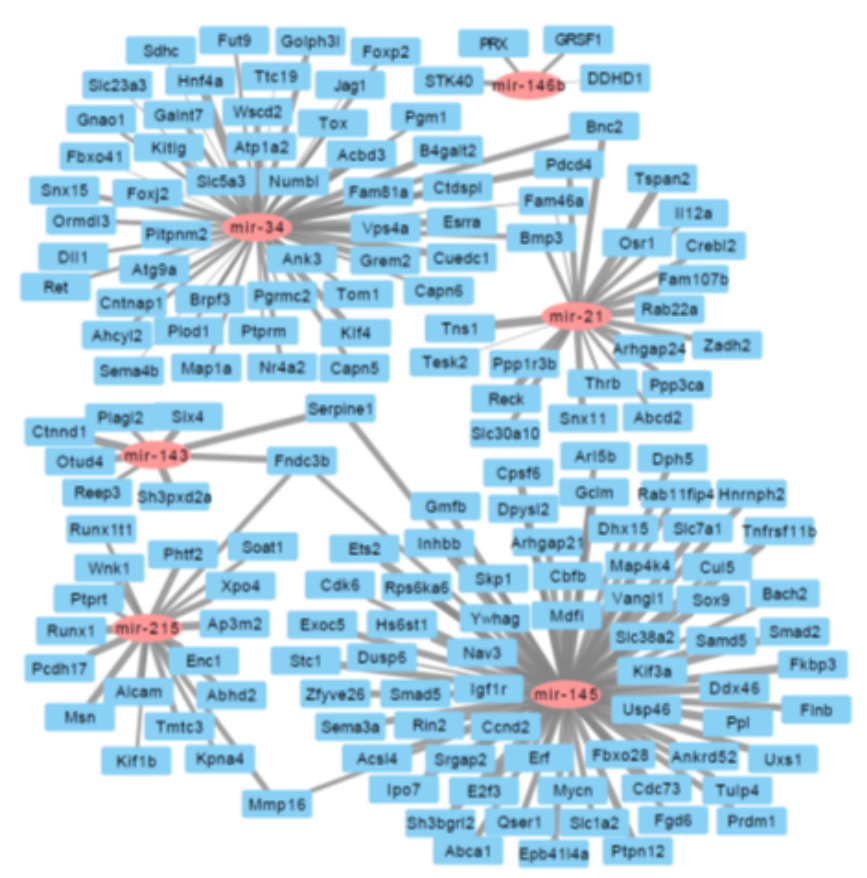

C WCN PCN PCT

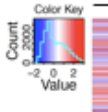

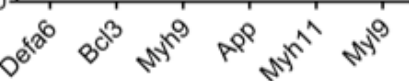

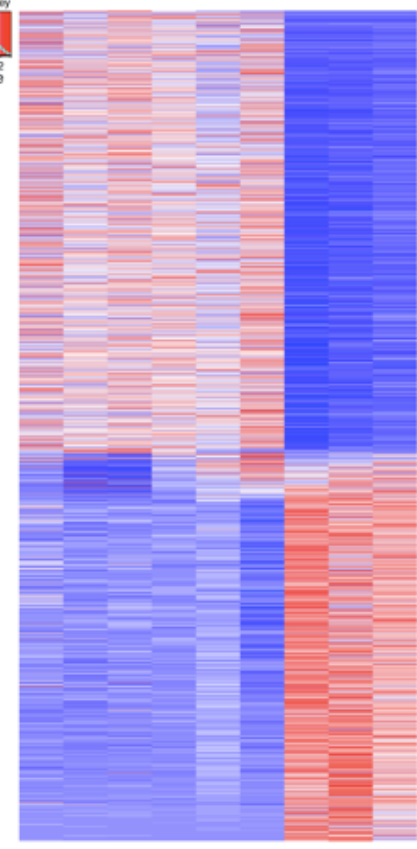

Figure 4 d Enriched Hallmark Genesets
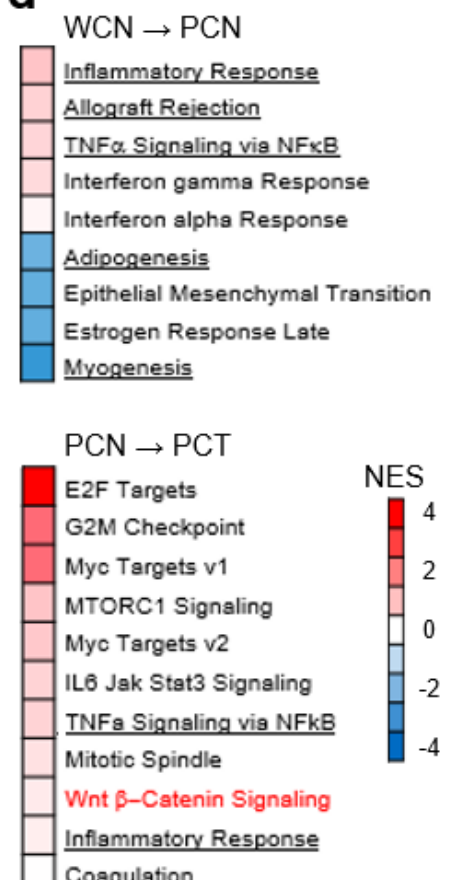

\begin{tabular}{|l} 
IL2 Stat5 Signaling \\
TGF beta Signaling \\
\cline { 1 - 1 } Unfolded Protein Response
\end{tabular}

$\square$ Complement

Allograft Rejection

Angiogenesis

Adipogenesis

Bile Acid Metabolism

Hypoxis

Oxidative Phosphorylation

KRAS Signaling Dn

Myogenesis

Underlined: incrementally altered $\mathrm{WCN} \rightarrow \mathrm{PCN} \rightarrow \mathrm{PCT}$

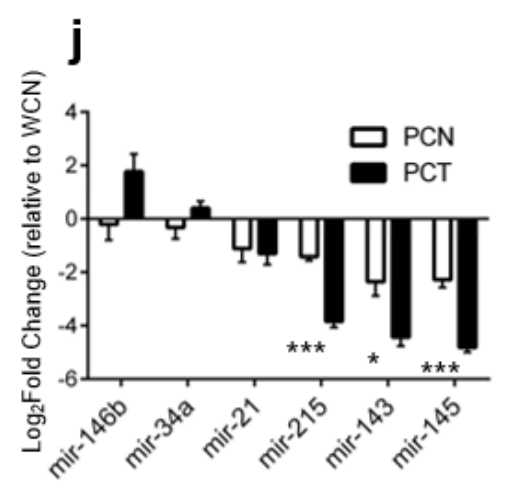


Transcriptomic analyses of colonic tissues obtained at 30-wks from Pirc and WT rats (no SPI treatment). (a) Principal component analysis (PCA) of RNA-seq data segregated tumor from non-tumor. WCN, WTcontrol diet-normal colonic mucosa; WSN, WT-SPI diet-normal colonic mucosa; PCN, Pirc-control dietnormal looking colonic mucosa; PCT, Pirc-control diet-colon tumor; PSN, Pirc-SPI diet-normal looking colonic mucosa; PST, Pirc-SPI diet-colon tumor. Each group contained three biological replicates, and Pirc normal looking colon was matched to Pirc colon tumor in the corresponding animal. (b) Venn diagrams summarizing differentially expressed genes (DEGs). (c) Heatmap of RNA-seq data representing all DEGs. (d) Enriched GSEA HALLMARK analysis of DEGs between WCN and PCN (upper panel), and between PCN and PCT (lower panel). Underlined terms indicate pathways altered incrementally for group comparisons: $\mathrm{WCN} \rightarrow \mathrm{PCN} \rightarrow \mathrm{PCT}$. Normalized enrichment score (NES) is indicated by color scale; -4 (blue) to +4 (red). Wnt signaling (red font) was one of several pathways enriched in the Apc-mutant model. (e) Validation by RT-qPCR of genes in five incrementally altered pathways, underlined in d). (f) Validation by RT-qPCR of genes related to tight junctions and antimicrobial function. (g) PCA of small RNA-seq data segregated tumor from non-tumor for miRNAs. (h) Heatmap of overall DEmiRs. (i) Cytoscape view of conserved miRNA-RNA targets and the predicted negative associations between RNA-seq and small RNA-seq data. (j) Experimental validation of selected miRNAs in Pirc colon tumors. ${ }^{*} p<0.05,{ }^{*} p<0.01,{ }^{*}{ }^{*} p<0.001$. Twotailed t-test was performed for RT-qPCR results in two-group comparisons. Numerical data are shown as mean $\pm S E M, n-3$.

a
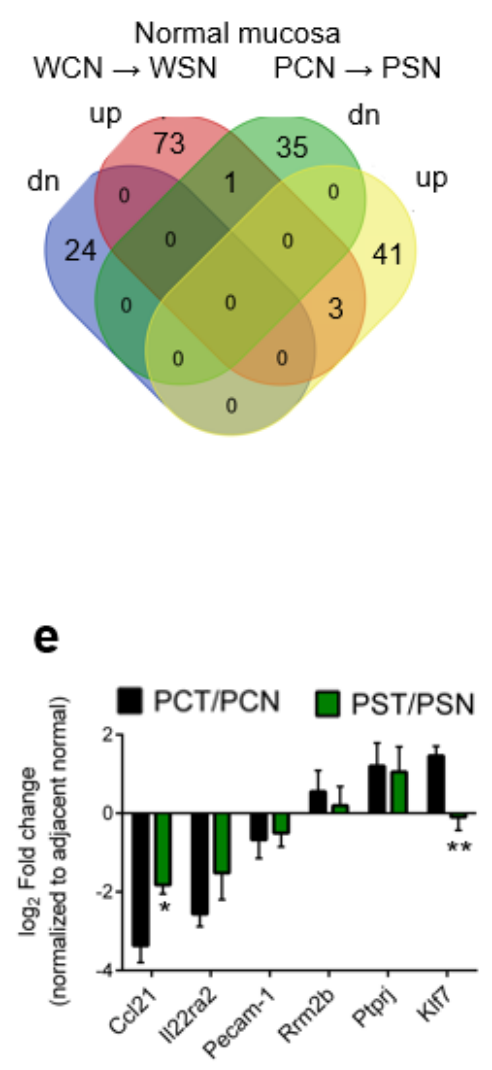

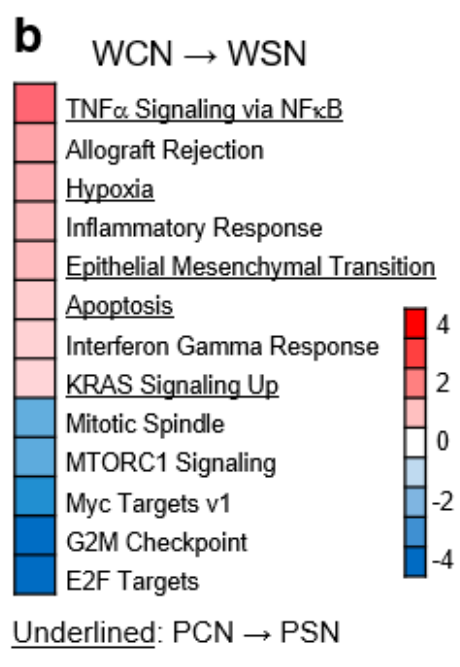

f

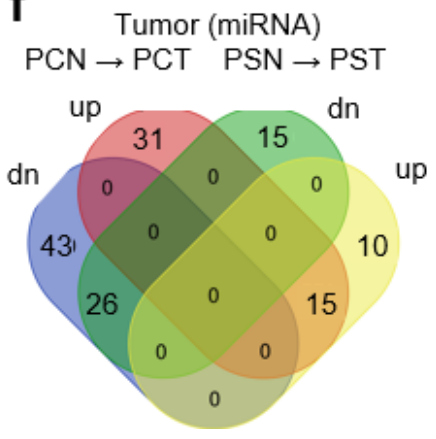

C $\mathrm{PCN} \rightarrow \mathrm{PCT} \stackrel{\mathrm{PSN} \rightarrow \mathrm{PST}}{\mathrm{P}}$
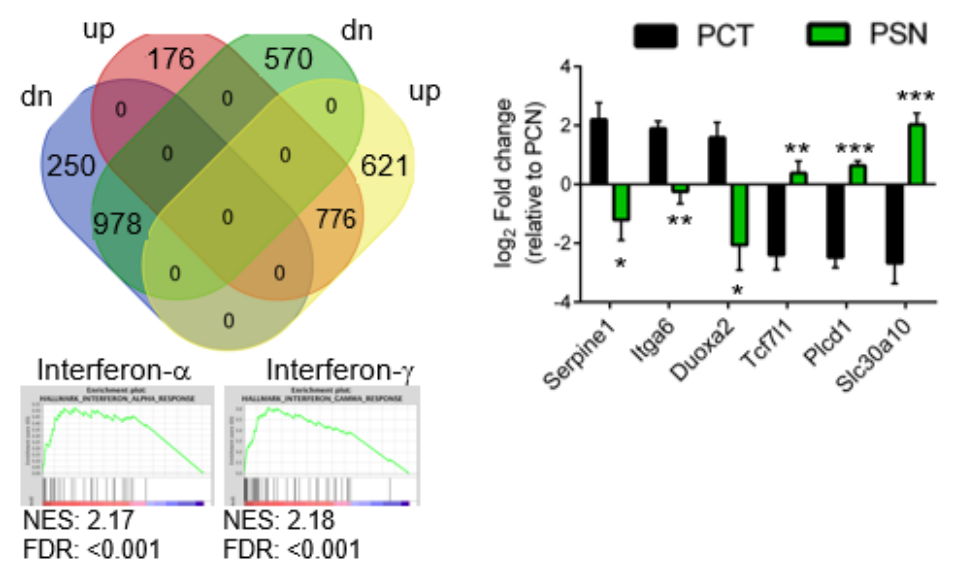

g

-PCN •PCT $\triangle$ PSN $\triangle$ PST
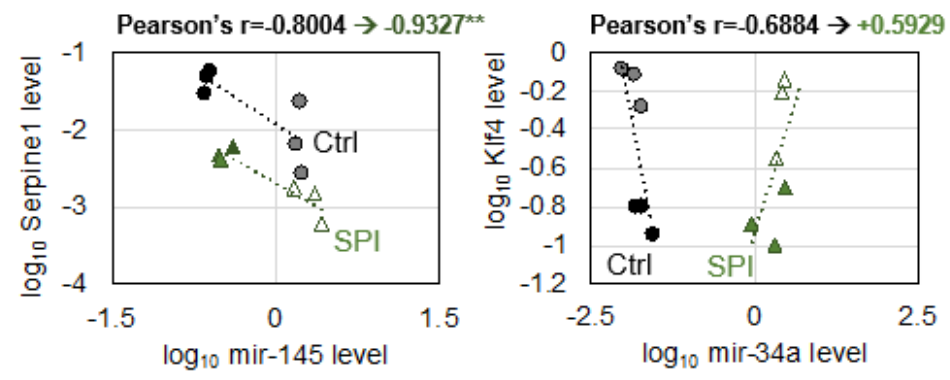

Figure 5 
Transcriptome changes due to SPI treatment. (a) Venn diagrams of DEGs for miRNAs altered by SPI treatment in Pirc and WT rats. (b) Statistically significant pathways from GSEA HALLMARK analysis, highlighting SPI effects on WT normal colon. Underlined terms indicate affected pathways in Pirc normal looking colon. (c) Venn diagram of tumor DEGs, comparing Pirc colon tumors after Ctrl or SPI diet consumption, and (below) GSEA enrichment in tumors arising despite SPI treatment (putative 'SPIresistant' colon polyps). (d) Validation by RT-qPCR of genes reversed in PCT vs. PSN groups. (e) Assessment of normalized molecular targets linked to oncogenic outcomes in Pirc colon tumors following SPI consumption. (f) Venn diagram of tumor DEmiRs in Pirc tumors from rats given Ctrl or SPI diet. (g) Corroboration of miRNA-mRNA relationships by RT-qPCR, in Pirc tissues from Ctrl and SPI treatment groups. ${ }^{*} p<0.05,{ }^{*} p<0.01,{ }^{* \star *} p<0.001$. Numerical data are presented as mean $\pm S E M, n=3$. For Pearson's correlation, degree of freedom $(\mathrm{N}-2)=4$ and the critical value $=0.917(\mathrm{p}=0.01)$. Table 7 in Additional file 3 provides miRNA-mRNA prediction data for miR145/Serpine1 and mir-34/KIf4.
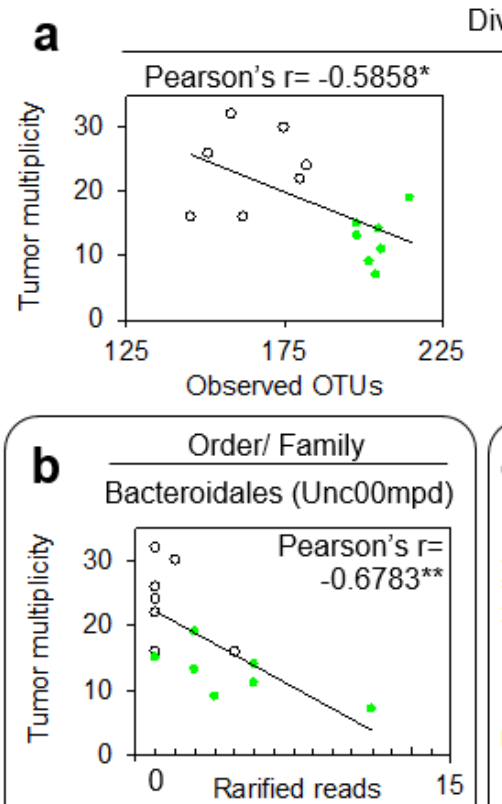

Bacteroidales (Unc01tc5)
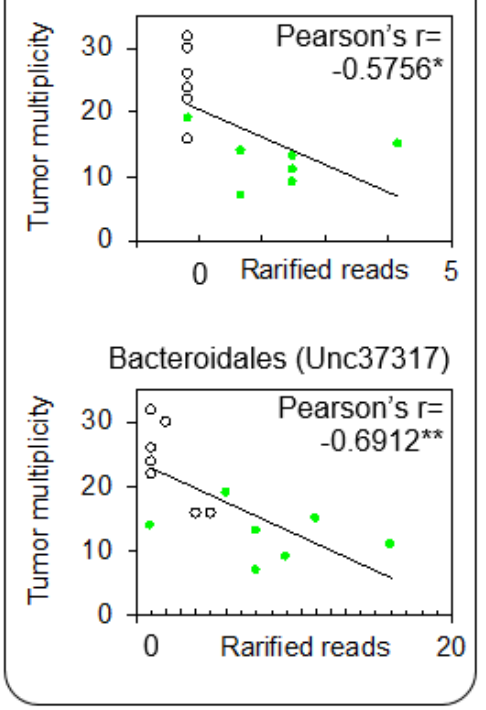

Diversity
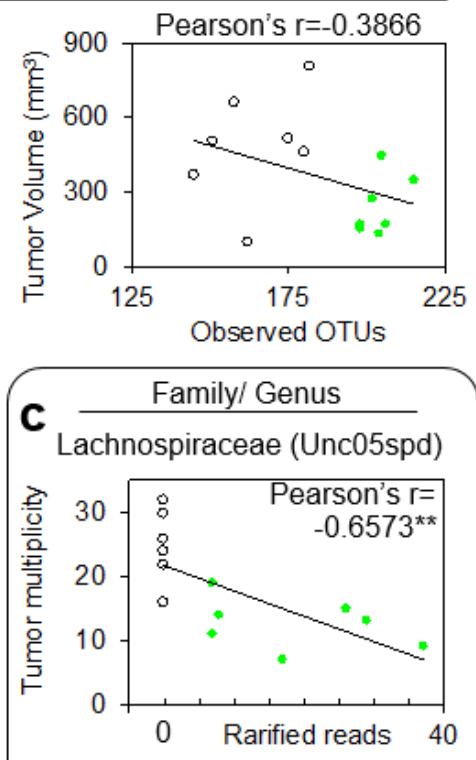

Ruminococcaceae (Unc04qod)

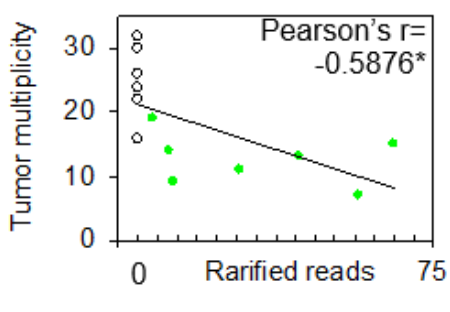

Ruminococcaceae (Unc45109)

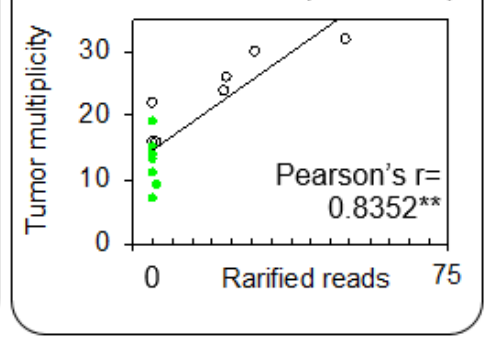

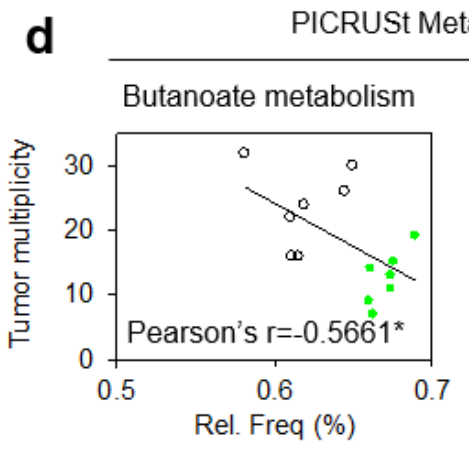

PICRUSt Metagenome Prediction

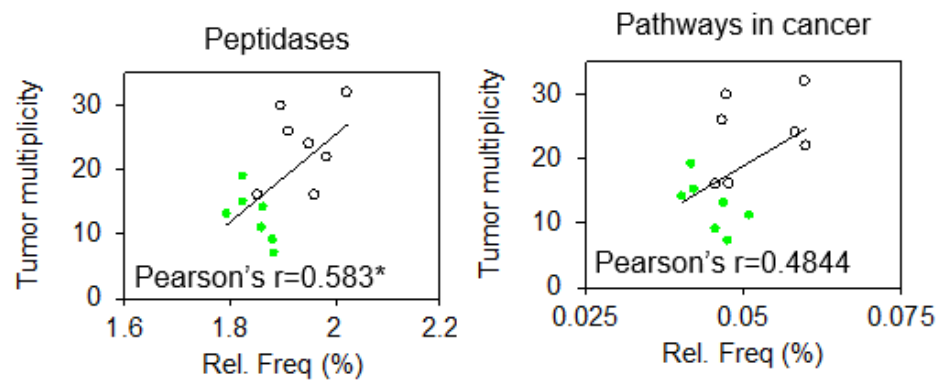

e

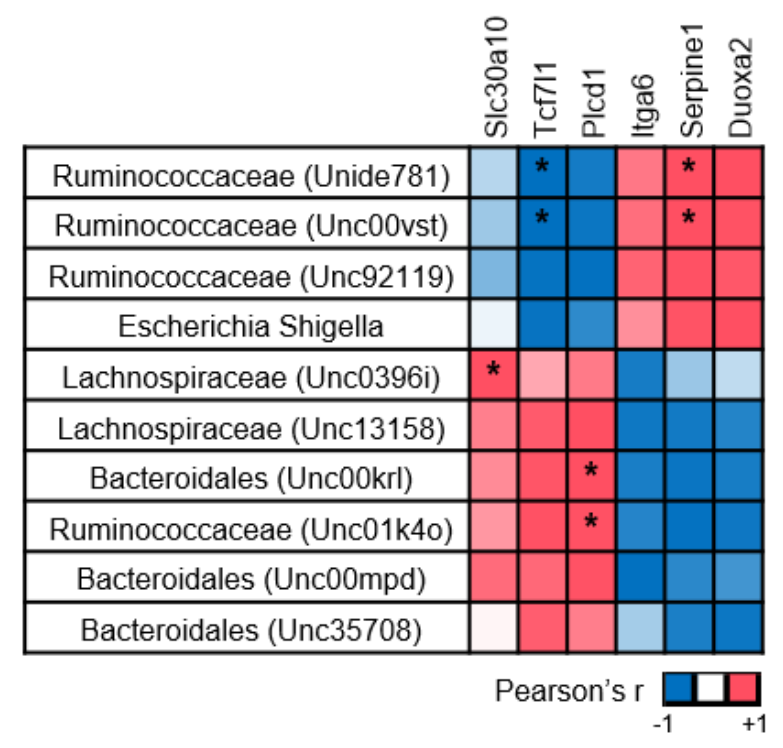

Figure 6 
Integrating gut microbiome, gene expression and tumor outcome data after SPI treatment in the Pirc model. (a) Associations between the gut microbiome diversity and host disease outcomes, expressed as tumor multiplicity or tumor volume. Associations also were probed between tumor multiplicity and the relative abundance of bacterial OTUs at the (b) family and (c) genus level. (d) Correlations between tumor multiplicity and metagenome prediction. (e) Associations between relative abundance of bacterial OTUs and anticancer/tumor suppressor genes identified in Pirc colon. Pearson's correlation coefficient data were represented as positive (red) or negative (blue); asterisk indicates statistical significance. For Pearson's correlation in $a)-d)$, degree of freedom $(\mathrm{N}-2)=12$ and the critical value $=0.533(p=0.05)$, and $0.661(p=0.01)$. For Pearson's correlation in $e)$, degree of freedom $(\mathrm{N}-2)=1$ and the critical value $=0.997$ $(p=0.05)$. 

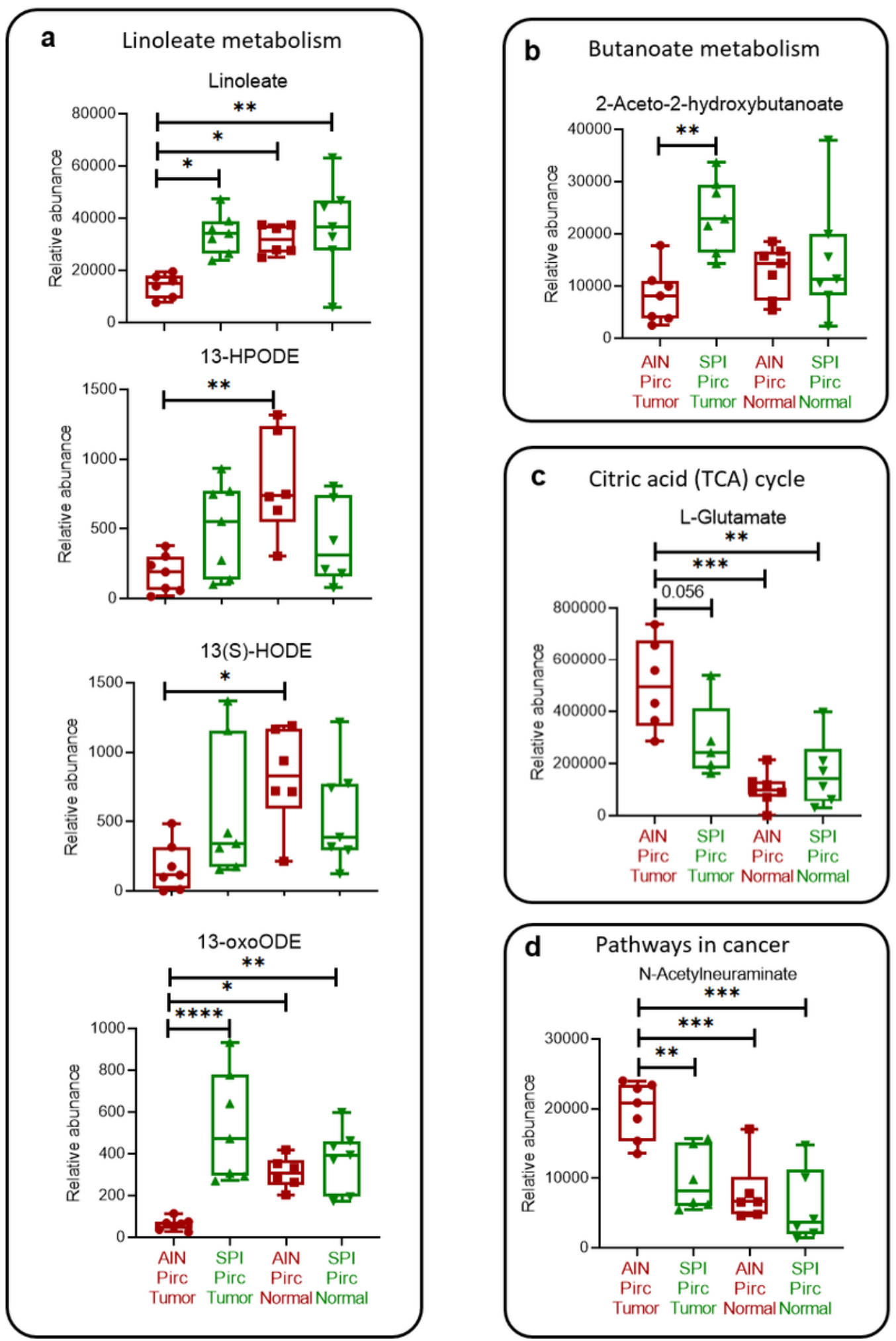

Figure 7

Metabolomic data pertaining to SPI consumption in the Pirc model. (a) Linoleate (linoleic acid) and its metabolites. (b) 2-aceto-2-hydroxybutanoate. (c) L-glutamate. (d) N-acetylneuraminate. Each datapoint represents one colon tumor or one normal colonic mucosa sample from rats in the corresponding groups. One-way ANOVA was used to compare the mean of each column with the mean of every other column, 
with Tukey correction for multiple comparisons (GraphPad Prism 9.0). ${ }^{\star} p<0.05 ;{ }^{\star \star} p<0.01 ;{ }^{* \star \star} p<0.001$; $\star \star \star \star x p 0.0001$.

\section{Supplementary Files}

This is a list of supplementary files associated with this preprint. Click to download.

- YSChen152021Additionalfile1.pptx

- YSChen152021Additionalfile2.pptx

- YSChen152021Additionalfile3.xlsx

- YSChen152021Additionalfile4.pptx

- YSChen152021Additionalfile5.pptx 